\title{
Subjekttheoretische Annäherungen an zeitgenössische Antifeminismen
}

\author{
Autorinnen-Kollektiv ,SUBJEKT ${ }^{\text {1 }}$
}

\section{EINLEITUNG}

Die von dem Projekt REVERSE analysierten Antifeminismen verweisen nicht nur auf bestimmte gesamtgesellschaftliche Entwicklungen und wissenschaftliche wie gesellschaftspolitische Auseinandersetzungen. Vielmehr drängen sich - angesichts der durchaus unterschiedlichen Resonanz-Räume für antifeministische Positionen auch Fragen nach den affektiven, emotionalen und daher subjektiven Voraussetzungen für die entsprechenden Polarisierungen in der Gesellschaft auf. Wie lassen sich z.B. die starken affektiven Aufladungen erklären, mit denen Themen rund um die Geschlechterverhältnisse verhandelt werden? Und wie lässt sich verstehen, dass anscheinend immer mehr Menschen für autoritäre Protest- und Politikformen ansprechbar sind? Gibt es bestimmte (konflikthafte) Erfahrungen oder Zumutungen, bestimmte Wahrnehmungsweisen, kurz: ein bestimmtes subjektives Erleben in Bezug auf gegenwärtige gesellschaftliche Entwicklungen und Zustände, an die die entsprechenden Strategien besonders gut andocken können?

Die Rede vom Subjektiven setzt ein Verständnis von Subjektivität (und damit auch des Subjekts) voraus, das häufig nicht weiter expliziert (oder gar kritisch reflektiert) wird. Mit diesem Beitrag wollen wir deshalb einige - durchaus unterschiedliche und auch unter uns streitbare ${ }^{2}-$ Lesarten des Subjekts als Denkangebote

1 Dem Autorinnen-Kollektiv ,Subjekt` gehören an: Susanne Maurer, Denise Bergold-Caldwell, Barbara Grubner und Helga Krüger-Kirn. (Susanne Maurer zeichnet für die Rahmung und das Quer-Denken der drei Lesarten sowie für die Endfassung des Beitragstextes verantwortlich.).

2 Für diese ,streitbare Auseinandersetzung d danken wir der $A G$ Subjekt, an der zeitweise auch Christopher Fritzsche und Marion Näser-Lather beteiligt waren. Unsere unterschied- 
formulieren, die für die Analyse der aktuellen Antifeminismen unseres Erachtens produktiv sind. Der Beitrag ist Resultat eines Austausch- und Diskussionsprozesses, in dem sich unser jeweiliges Denken des Subjekts auch verändert hat. In den gegenseitigen Verstehensversuchen haben wir sowohl Annäherungen erfahren, als auch die Notwendigkeit klärender Unterscheidung und Abgrenzung. Wir haben bewusst nicht versucht, die dabei erkennbar werdenden Subjekt-Verständnisse in eine Gesamt-Systematik zu integrieren; vielmehr bleiben bestimmte Unvereinbarkeiten bestehen, die den Gegenstand Subjekt insgesamt sperrig (und damit auch widerspenstig?) halten.

Wir verfolgen also verschiedene Spuren, um zu reflektieren, was Menschen für antifeministische Positionen ansprechbar macht, und formulieren in diesem $\mathrm{Zu}$ sammenhang auch gesellschaftstheoretische Überlegungen zu der Frage, auf welche Art und Weise Menschen heute zu Subjekten werden. Für ein Verständnis davon, welche Dimensionen dabei Berücksichtigung finden sollten und wie diese wiederum miteinander verknüpft sind, entwickeln wir unterschiedliche Lesarten im Anschluss an Michel Foucault, an Sigmund Freud und an Jacques Lacan. Deutlich wird mit Bezug auf Foucault die jeweilige Subjektposition in (machtvollen und herrschaftlichen) Diskursen, mit Freud die (unbewusste) Psychodynamik und mit Lacan das (unbewusste) Subjekt des Begehrens. Jede dieser Lesarten birgt ein bestimmtes (und zugleich begrenztes) Erklärungspotenzial für die Frage nach den subjektiven Voraussetzungen für antifeministische Mobilisierungen. So lässt sich z.B. eine - wie auch immer ausgearbeitete und ausbuchstabierte - psychoanalytische Perspektive nicht ohne Weiteres auf ein Denken in Anlehnung an Foucault beziehen, auch wenn ein solcher Versuch immer wieder gemacht wird.

Nach einer Hinführung zur Rede vom Subjekt wird in diesem Beitrag als erste Lesart (vertreten und formuliert von Denise Bergold-Caldwell) von einer machtanalytischen Perspektive in Anlehnung an Foucault Gebrauch gemacht. Wir steigen bewusst mit dieser Perspektive ein, weil daran im Kontext geschlechtertheoretischer Reflexionen bereits vielfach angeschlossen worden ist. Das Subjekt mit Foucault zu denken, führt hier nicht zuletzt zu den Gouvernementalitäts-Studien, mit denen das Verhältnis von Fremd- und Selbstführung(en) in den Blick gerät. Die zweite Lesart (vertreten und formuliert von Helga Krüger-Kirn) stützt sich auf eine psychodynamisch geprägte feministisch-psychoanalytische Perspektive, die insbesondere auch den Körper zu theoretisieren sucht. Hier wird das Subjekt (sowie die Geschlechterdifferenz) als Schnittstelle zwischen Individuum und Gesellschaft gesehen und mit komplexen psychischen Strukturen zusammengedacht, die ihren Ausgangspunkt in

lichen theoretischen (und auch fachlichen) Bezüge haben wir als bereichernd erlebt. In unserem Beitrag zollen wir dieser Heterogenität nicht zuletzt durch die Formulierung ,Lesarten` Respekt. 
der Verarbeitung infantiler Wünsche, Erfahrungen und Problemlösungen haben. Die dritte Lesart (vertreten und formuliert von Barbara Grubner) bezieht sich auf eine feministische Position, die vom Spätwerk Lacans inspiriert ist und damit eine nochmals andere Wendung psychoanalytischer Denkmöglichkeiten ins Spiel bringt. Die Neuakzentuierung von Subjekt, Geschlecht und Begehren des späten Lacan wird für die Formulierung feministischer Gegenwartsdiagnosen aufgegriffen und für die Frage produktiv gemacht, warum autoritäre Politik- und Protestformen derzeit erstarken oder genauer: was Subjekte für diese Angebote und Entwürfe überhaupt zugänglich macht.

\section{ZUR Rede Vom SUbJeKT}

Inwiefern unterscheidet sich die Rede vom Subjekt von der Rede über das Individuum oder die Person, in gewisser Weise auch von der Rede über Akteur_innen ${ }^{3}$ ? Zunächst kann festgehalten werden, dass die Rede vom Subjekt vor jeweils differenten disziplingeschichtlichen und fachkulturellen Hintergründen und zudem im Hinblick auf unterschiedliche Erkenntnisanliegen und Aufgabenstellungen erfolgt (vgl. Zima 2017); das hat jeweils komplexe subjektphilosophische Implikationen. Wenn von einem menschlichen Subjekt gesprochen wird, so geht es in sozial- und geisteswissenschaftlicher Perspektive, verkürzt gesagt, um die Möglichkeit der Selbstbezüglichkeit, des Selbstbewusstseins sowie des Handelns und Agierens als Selbst. (Subjekt-)Kritische Beiträge verweisen allerdings darauf, dass das Subjekt nicht als ein ,eigentliches' Selbst gedacht werden kann, sondern in Bezug auf sein Handeln, seine Begehrensweisen und Seinsweisen immer als Schnittstelle in/zur Gesellschaft gedacht werden muss. Solchen Lesarten des Subjekts sehen wir uns in diesem Beitrag verpflichtet, wenn wir einerseits herausstellen, wie das Subjekt machtanalytisch gedacht werden kann, und wie es andererseits mithilfe verschiedener gesellschaftstheoretisch-psychoanalytischer Betrachtungsweisen zu fassen wäre.

Im Rahmen der folgenden Überlegungen denken wir Subjekt in Abgrenzung zur klassischen Subjektphilosophie, die ein erkennendes, selbst-bewusstes und Sinnstiftendes Subjekt (als zentriertes Subjekt) quasi voraussetzt. Vielmehr beziehen wir uns auf Überlegungen, die ein dezentriertes Subjekt konzeptualisieren ${ }^{4}$ - so etwa im

3 Wir verwenden in unserem Beitrag grundsätzlich den Gender-Gap, um damit das Denken der Zweigeschlechtlichkeit zu öffnen und zu durchkreuzen. Zugleich werden wir aber auch von ,Männern' und ,Frauen“ als vergeschlechtlichten Gruppen und von ,männlich“ und ,weiblich`als Attribuierungen sprechen.

4 Vgl. als Variante hierzu die Vorstellung von Subjekt als ,zentrierte Vielfalt“ bei Maurer (2001, 2013). 
Kontext von Strukturalismus und Poststrukturalismus, aber auch im Kontext der Psychoanalyse. Dabei eröffnen die Denkmöglichkeiten, wie sie etwa im Anschluss an Foucault, Freud und Lacan entwickelt worden sind, jeweils deutlich andere Sichtweisen: Mit Foucault werden zum einen (mögliche und konkret eingenommene) Subjekt-Positionen in den Gefügen der Macht beschreibbar, zum anderen lassen sich je spezifische (historisch-gesellschaftlich bedingte bzw. ermöglichte) Subjektivierungsweisen rekonstruieren; mit Freud wird das Subjekt als Subjekt des Unbewussten lesbar und mit Lacan als unbewusstes Subjekt des Begehrens.

Foucaults Versuche, das Subjekt zu fassen, bringen dessen Subjektivierung über Macht-Wissens-Diskurse in den Fokus, während Freud vor allem dessen Unbewusstes und die damit verbundene - auch leiblich-körperlich vermittelte - Psychodynamik verdeutlicht; Lacan wiederum nimmt eine sprachtheoretische Grundlegung des (unbewussten) Subjekts des Begehrens vor. ${ }^{5}$ Wie auch immer konzeptualisiert - im Zuge seiner Dezentrierung hat das Subjekt ,seinen Ort als Null- und Fixpunkt des philosophischen und humanwissenschaftlichen Vokabulars [...] [verloren], es erweist sich [...] [selbst] in seiner Form als abhängig von gesellschaftlich-kulturellen Strukturen, die ihm nicht äußerlich sind“" (Reckwitz 2008: 13). So unterstreichen denn auch die in diesem Beitrag diskutierten Lesarten, dass das Subjekt von Beginn an gesellschaftlich gedacht werden muss.

Subjekt wird damit gewissermaßen als Ort verstanden, in und an dem sich Gesellschaft artikuliert. Das heißt, dass gesellschaftliche Strukturen zugleich ermöglichende wie begrenzende Bedingungen sind, in und unter denen das Subjekt hervorgebracht wird. Um das zu verdeutlichen, beziehen wir uns im Folgenden auf Denktraditionen und Denkangebote, die genau an dieser Schnittstelle zwischen Individuellem und Gesellschaftlichem arbeiten. Unsere gemeinsamen Denk-Bewegungen zwischen den unterschiedlichen Lesarten des Subjekts insistieren dabei auf eine Perspektive, die ,das Wechselverhältnis zwischen gesellschaftlichen Strukturen/Diskursen/Zwängen und der individuellen Herausbildung und Struktur einer lebenslang konflikthaften Subjektivität nicht in eine Richtung auflöst.“ (Bereswill 2018: 32) Eine solche Perspektive ist insbesondere dort notwendig und auch weiterführend, wo affektive Motive auf mehr oder weniger verborgene und unterdrückende (Macht-)Interessen verweisen, wie im Fall antifeministischer Artikulationen und Ressentiments. Die Denk-Bewegung zwischen Psychoanalyse und Machtanalytik ermöglicht ein Verständnis des Subjekts, das dessen Situiertheit in gesellschaftli-

5 Deshalb hat die Rede von Subjektivierung im Kontext der drei Lesarten auch jeweils unterschiedliche Bezugspunkte (die auch innerhalb der verschiedenen theoretischen Felder noch kontrovers verhandelt werden). Was den hier verfolgten Lesarten des Subjekts jedoch gemeinsam ist, ist ein Moment der Unverfügbarkeit des Subjekts, was sie für (gesellschafts-)kritische Reflexionen interessant macht. 
chen Kräftefeldern ebenso in Betracht zieht wie die Bedeutung und Wirkmächtigkeit von Affekten. Das Subjekt wird damit als komplexes (sprachlich-leiblich-körperliches) Gebilde erkennbar, das auch Präverbales umfasst und das ohne libidinöse Antriebspotenziale, affektive Ausdrucksformationen und diskursive Ermöglichungen und Einschreibungen nicht zu denken ist.

\section{Foucault Und die Frage des SubJekts}

Sich auf Michel Foucault beziehende Subjekt-Verständnisse adressieren das Subjekt vor allem über die Frage der Subjektivierung. Subjektivierung wird hier - im Unterschied zu den beiden psychoanalytischen Lesarten - als Unterwerfung des Subjekts unter Macht-Wissens-Ordnungen verstanden, die ein Subjekt als solches erst hervorbringen. Neben einem begrifflichen Instrumentarium (u.a. Diskurs, Dispositiv, Selbsttechniken) und den entsprechenden theoretischen Perspektiven beinhaltet eine Foucault'sche Herangehensweise die Frage nach den unterschiedlichen Machtprinzipien (oder Modalitäten der Macht), die in diesen Wissensordnungen evident werden. In Bezug auf die Frage, warum und wie antifeministische Mobilisierungen bei den Subjekten ,ankommen' (bzw. an ihr subjektives Erleben andocken können), lassen sich mit der Foucault'schen Werkzeugkiste unterschiedliche oder auch keine - Antworten finden.

Die Frage der Affektivität, Emotionalität oder der ,psychischen Verankerung ${ }^{c}$ wird in dieser Lesart in erster Linie über die Machtprozeduren analysiert, die solche Emotionen hervorbringen könn(t)en. Mit Foucault lässt sich ein spezifischer Blick auf Prozesse der Führung von Subjekten richten - und darauf, wie diese Subjekte diese Führung als Selbstführung übernehmen und sich als ein Selbst erlernen (Ricken 2013: 29), aber nicht, wie diese Prozesse ihre psychisch-affektive Verankerung erfahren (vgl. Butler 2001). Das (bewusstseinsphilosophisch gedachte) Subjekt der Erkenntnis war für Foucault der Ausgangspunkt für seine kritische Auseinandersetzung mit der Hervorbringung des Subjekts in Macht-Wissens-Diskursen (Reckwitz 2008). Es ging ihm darum, historisch-genealogisch zu rekonstruieren, wie Menschen in , unserer ${ }^{\star}$ Kultur zu Subjekten werden (Saar 2013) und damit die epistemische (Dimension der) Subjekt-Genese zu markieren - sie also von der ontologischen Subjekt-Genese abzugrenzen, und so auch von der Konzeption einer Innerlichkeit, einer (vor-epistemisch gedachten) unbewussten Sexualität, die es zu befreien gilt. Foucaults Blick fängt die Logiken der Hervorbringung spezifischer Subjektpositionen ein. Er verdeutlicht durch seine konkreten Untersuchungen, wie sich Menschen als ein solches bzw. als ein bestimmtes Subjekt erkennen.

Theoretiker_innen wie Lemke (2000), Saar (2013) oder Bublitz (2014) teilen Foucaults Werk in unterschiedliche Schaffensperioden ein und machen in diesen 
Perioden auch unterschiedliche Zugriffe auf das Subjekt aus. Nach einem kurzen Rekurs darauf kommt es uns vor allem darauf an, zu verdeutlichen, welche Denkangebote Foucault'scher Provenienz uns darin unterstützen können antifeministische und autoritäre Politiken zu reflektieren. Es sind insbesondere zwei Analyse-Werkzeuge, die uns hier besonders fruchtbar erscheinen: das Nachdenken über die Gouvernementalität der Gegenwart und die damit adressierbaren neoliberalen Selbsttechniken (Foucault 2007; Bröckling/Krasmann/Lemke 2007), sowie die Analyse des gegenwärtigen Sexualitätsdispositivs (Dietze 2016, 2017), das in Verbindung mit der Gouvernementalität der Gegenwart zu sehen ist.

\subsection{Etappen des Foucault'schen Nachdenkens über das Subjekt}

Foucaults erste Auseinandersetzung mit dem Subjekt beschäftigt sich vor allem mit den Ausschlussmechanismen, die in der abendländischen Kultur ein spezifisches Subjekt hervorgebracht haben (Keller 2011). Über die Abgrenzung und Kategorisierung der ,Wahnsinnigen', der ,Kranken', der ,Delinquenten“ und über eine historisch spezifische Perspektive auf den Tod wird demnach ein besonderer Subjekttypus hervorgebracht (ebd.). Angesprochen ist damit zweierlei: Zum einen das abendländische Disziplinar-Subjekt, und zum anderen die Ordnung des (abendländischen, wissenschaftlichen) Diskurses, der auf die Hervorbringung des Subjekts der Erkenntnis (und entsprechender Subjektpositionen) eingewirkt hat. So macht Foucault deutlich, dass beispielsweise Delinquent_innen erst im Rahmen einer historischspezifischen diskursiven Ordnung als solche in Erscheinung treten, also über spezifische (An-)Ordnungen zu einem Subjekt der Delinquenz werden. Foucaults Analysen verdanken wir die Erkenntnis, dass sich die Macht-Wissens-Ordnungen auch und insbesondere in die Körper der Subjekte einschreiben. Bereits im Anschluss an seine frühen Studien Wahnsinn und Gesellschaft (Foucault 1969) und Die Geburt der Klinik (Foucault 1973), aber spätestens im ersten Band seines Werkes Sexualität und Wahrheit (Foucault 2013) stellt er resümierend fest, dass sein bislang verwendeter Machtbegriff $z u$ juridisch ${ }^{6}$ gedacht ist, und er verlagert sein Interesse darauf, den Machtbegriff zu erweitern.

6 Der Begriff juridisch verweist hier auf ,feste Normenstrukturen', wie sie - quasi als Ausdruck bestimmter Macht- und Herrschaftsverhältnisse z.B. in Gesetzen gefasst sind. Der Begriff geht über den (deutschen) Begriff ,juristisch` hinaus, weil letzterer sich nur auf juristische Rechtsstrukturen bezieht. Das macht u.a. Isabel Lorey (2017) in ihrer kritischen Auseinandersetzung mit dem juridischen Machtbegriff von Judith Butler deutlich. Lorey weist darauf hin, dass ,juridisch ' bei Butler auch das ,Gesetz des Vaters' im Sinne der Lacan'schen Psychoanalyse umfasst. Zugleich ist die Position Butlers von der Foucaults zu unterscheiden: Während für Butler ,,[d]er Begriff der juridical structures [...] ei- 
Als nächster Analyseschritt wird in der Foucault-Rezeption häufig die Hinwendung zum Begriff des Macht-Diskurses markiert (Dreyfuss/Rabinow 1994). Mit Bezug auf den Diskurs erscheint z.B. der ärztliche Blick, wie er in Die Geburt der Klink (Foucault 1973) beschrieben wurde, nicht mehr allein an die Person des Arztes gebunden (und damit in gewisser Weise ,ursprünglich'), vielmehr nimmt der Arzt lediglich eine spezifische Position im Diskurs der Klinik ein, die es ihm ermöglicht zu sprechen und seine Wirkung als sogenannter Experte zu entfalten. Mit dieser Wendung erweitert Foucault also seinen Macht-Begriff; anstatt einer juridischen Machtkonzeption wird die Diskursivität der Macht in den Mittelpunkt der Analyse gestellt. Spätestens in Sexualität und Wahrheit (Band 1) warnt Foucault (2013) gar davor, Macht einzig und allein als Repression zu verstehen, vielmehr müsse auch ihre Produktivität in Analysen mit einbezogen werden. Mit seinem Konzept der Bio-Macht zeigt er beispielsweise auf, inwiefern Episteme, Dispositive und Diskurse Sexualität (als spezifisches Konzept) erst hervorgebracht haben und dass es keine ,Innerlichkeit‘ und keine ,Ursprünglichkeit‘ (also keine Essenz) der Sexualität gibt. Viele feministische und postkoloniale Analysen schlossen genau hier an. ${ }^{7}$

Foucault (1993) wendet sich nun den Technologien des Selbst zu - als Techniken, die ,das Selbst' anwendet (auch mithilfe anderer), um sich selbst zu verbessern, glücklicher zu sein - oder einfach Veränderungen herbei zu führen. Diese Techniken werden z.B. in Form von Körperpraktiken, rituellen Wiederholungen oder auch Bewusstseinsübungen vollzogen. Christoph Menke (2003) beschreibt sie insgesamt als Übungen. Die Übungen haben laut Menke sowohl einen befreienden als auch einen unterwerfenden Charakter. Foucault (2001) formuliert diese Perspektive in seiner Vorlesung Hermeneutik des Subjekts, in der er historisch untersucht, wie sich die Technologien des Selbst in ,unserer' Kultur historisch entwickelt haben. Mit seiner Vorlesung zur Gouvernementalität (Foucault 2003) können die ,Technologien des Selbst' (oder auch ,Selbsttechniken`) in Zusammenhang mit

ner der zentralen Begriffe [ist] [...] ohne den die Funktion anderer Begriffe wie ,Macht", ,Gesetz', ,Diskurs', ,Sprache‘ und ,Subjekt‘ nicht verständlich [wird]“, bedeutet ,juridisch“ in einer Foucault'schen Perspektive ,eine spezifische Positionierung dahingehend [...], wie Macht und Herrschaft verstanden werden.“ (Lorey 2017: 49) In ihrer reflexiven Auseinandersetzung mit Butlers theoretischem Zugang argumentiert Lorey, dass Butlers juridische Machtkonzeption nicht zuletzt deshalb fehlgeht, weil das Gesetz der Norm von ihr nicht historisiert wird (Lorey: 72).

7 So etwa Bührmann (1995) und Lorey (2017) im Kontext der feministischen Theorie und der Gender Studies, Stuart Hall (1996) im Kontext der cultural studies (u.a.) mit Bezug auf Rassismus, und Edward Said (1979) im Kontext der postcolonial studies, nicht zuletzt mit Bezug auf die Konstruktion des ,Orients'. 
Techniken der Führung und des Regierens betrachtet werden. GouvernementalitätsStudien (u.a. Lemke/Krasmann/Lemke/Bröckling 2007) rekonstruieren nicht zuletzt über den Begriff des „Unternehmerischen Selbst“ (Bröckling 2007), wie bestimmte Techniken der Führung im Kontext neoliberaler Gesellschaftsentwürfe in ,Selbstführungen` und Selbsttechniken übernommen werden.

Anders als Ansätze, die sich auf die Gouvernementalität der Gegenwart beziehen, sehen einige Autor_innen in der Hinwendung zum Selbst aber auch eine ethische Wende bei Foucault (so z.B. Schmid 2002). In der feministischen Theoriebildung überwiegt jedoch der Anschluss an die Gouvernementalitäts-Studien, wobei hier zugleich auch Fragen der Transformation des Subjekts theoretisiert werden (vgl. hierzu Bublitz 2014). Diese Perspektive ermöglicht es, die Selbsttätigkeit der Subjekte zu betrachten, sie aber nicht losgelöst von Macht-Wissens-Ordnungen zu sehen. Wir möchten daher in Bezug auf die Frage, warum und wie antifeministische Diskursproduktionen ihre Verankerung in den Selbstbezügen der Subjekte finden, eine Lesart ins Spiel bringen, die von einer Veränderung im Sexualitätsdispositiv ausgeht und dieses mit den spezifischen gouvernementalen Subjektivierungen spätmoderner Subjekte in Verbindung bringt.

\subsection{Antifeminismen im Kontext des spätmodernen Sexualitätsdispositivs und der Gouvernementalität der Gegenwart}

Antifeministische Diskursproduktionen und Akteur_innen beziehen sich zentral auf Geschlechterkonfigurationen und - zumindest immanent - auch auf die diskursiven Hervorbringungen von Sexualität. Gabriele Dietze $(2016,2017)$ sieht in der momentanen Diskursivierung des Sexes ein spätmodernes Sexualitätsdispositiv am Werk, das die Verschränkungen unterschiedlicher Macht- und Affektblöcke (hier: Sexismus und Rassismus) ermöglicht. Sie leitet ihre Konzeptualisierung des spätmodernen Sexualitätsdispositivs von Foucault ab, hebt aber - in sexismus- und rassismuskritischer Perspektive - hervor, dass sich dieses Dispositiv durch die Gewährung von Freiheiten unter Hervorhebung der positiven Norm der ,bereits existierenden Geschlechtergerechtigkeit in westlichen Ländern` auszeichnet:

„Wir sehen einen autochthonen [...] Diskurs, der für sich in Anspruch nimmt, die sexuelle Befreiung bereits vollendet zu haben, Frauen enthysterisiert, adoleszente Sexualität anerkannt und Homosexuelle normalisiert zu haben. Ins Zentrum gerückt ist ein Phantasma der Gewährung, der Säkularität, der Freiheit und des Willens und der Fähigkeit abendländischer Bürger, von diesen ,Freiheiten“ Gebrauch zu machen.“ (Dietze 2016: 99) 
Das Spezifische des bürgerlichen Sexualitätsdispositivs im 18., 19. und beginnenden 20. Jahrhundert bestand darin, über einen deklarierten ,sexuellen Notstand“ ein Netz von Bedeutungen und Diskursen über sämtliche Aspekte der Sexualität hervorzubringen. Auf diese Weise konnte disziplinierend auf die Menschen eingewirkt werden, ohne ihnen das Gefühl entwenden zu müssen, dass sie sich auf dem Weg der voranschreitenden sexuellen Befreiung befänden. ${ }^{8}$

Im Kontext des spätmodernen Sexualitätsdispositivs scheint es nun nicht mehr um eine Normierung der Sexualität unter den Bedingungen ihrer Diskursivierung zu gehen; vielmehr funktioniert das aktuelle Sexualitätsdispositiv über den „Imperativ der Gewährung und Freiheit“ (Dietze 2016: 100). Dietze hebt hervor, dass dieses Sexualitätsdispositiv zudem über die Behauptung einer geschlechterpolitischen Sonderstellung zur Geltung gebracht wird. Damit ist gemeint, dass eine als bereits vollzogen unterstellte Frauenemanzipation bzw. eine als bereits erreicht unterstellte Geschlechtergerechtigkeit gegenüber ,Anderen' (die in dieser Hinsicht als ,rückschrittlich ' imaginiert und markiert werden), verteidigt' und ,bewahrt ${ }^{\text {‘ werden }}$ muss.

Diesen Gegenpol der ,Anderen“ bilden heute insbesondere muslimische Migrant_innen und Geflüchtete, deren ,Kultur' und Sozialisation sie angeblich jenseits der liberalen Freiheit positionieren - das zeigt besonders die Fallstudie Ethnisierung von Sexismus im Rahmen des REVERSE-Projekts (Bergold-Caldwell/Grubner in diesem Band), einige Hinweise finden sich aber auch in den Befunden der Fallstudie Sexualpädagogik (Oldemeier et al. in diesem Band). Neben der medial besonders intensiv verhandelten ,Figur des jungen muslimischen Mannes‘ sollte an dieser Stelle nicht vergessen werden, dass zur Aufrechterhaltung dieser Konstruktion auch die ,nicht-emanzipierte muslimische Frau' - zumindest implizit - stets mit-imaginiert wird (vgl. Bergold-Caldwell/Grubner 2017).

Mit der Hinwendung zu der hier mit Bezug auf Dietze gekennzeichneten, neuen (spätmodernen) Form des Sexualitätsdispositivs hat sich nicht nur die Regierung des Sexes verändert; vielmehr muss diese Erkenntnis auch in Zusammenhang mit insgesamt veränderten Rationalitäten betrachtet werden. Hier kommt erneut die Perspektive der Gouvernementalitäts-Studien ins Spiel.

8 Die Negativfolie war hier das Proletariat: Da es lange Zeit als unfähig galt, seine Triebe unter Kontrolle zu halten, war es weitaus weniger strikten Tugendregimen unterworfen als das Bürgertum (Gehring 2014). Auch Menschen in den damaligen Kolonien und rassifizierte Andere entsprachen dieser Negativfolie. Stoler (1995) fordert, Foucault diesbezüglich zu ergänzen; bereits im 18. Jahrhundert galt der ,Schwarze Mann“ als sexuell hyperaktiv, während die Schwarze Frau als entweder von ihm bedroht oder aber selbst als exotisch sexualisierte Andere diskursiviert wurde (ebd.). 


\subsection{Das Subjekt zwischen Fremdführung und Selbstführung}

Wie bereits angemerkt, untersuchen die Studien zur Gouvernementalität die Rationalität der Führung insbesondere mit Bezug auf das Verhältnis von Fremd- und Selbstführung (und deren jeweilige Übersetzungen ineinander). In seinen Vorlesungen zur Gouvernementalität erörtert Foucault (2003) eine Veränderung in den Regierungsformen bzw. Regierungsmodalitäten des Staates: Im 17. und 18. Jahrhundert wurden Texte zur Regierungskunst veröffentlicht, die eine Regierung des Staates analog zur ,Regierung einer Familie“ nahelegen. In diesem Zusammenhang tritt die Vorstellung des Herrschers als Souverän zurück gegenüber dem Leitprinzip einer ökonomischen Führung, ,die als Hauptzielscheibe die Bevölkerung, als Hauptwissensform die politische Ökonomie und als wesentlich technisches Element die Sicherheitsdispositive hat." (Foucault 2003: 820) Diese neue Weise des Regierens im Liberalismus folgt keiner repressiven Logik mehr, es entsteht vielmehr „eine Form der Macht, die nicht über Zwang, Gesetze oder Repression operiert, sondern über die ,Führung der Führungen': Über das Abstecken eines Möglichkeitsfeldes, auf dem sich Menschen gemäß bestimmter Deutungsformen selbst führen.“ (Ludwig 2019: 2)

Diese historisch bereits früh formulierte Gestalt(-ung) der Macht(-ausübung) wird ab den 1970er Jahren zu einer zunehmend sich ausprägenden Tendenz. Viele Sozialwissenschaftler_innen bringen diese Tendenz mit dem Aufstieg des Neoliberalismus infolge der Krise des Fordismus in Zusammenhang. Aus einer Foucault'schen Perspektive lässt sich hier von einer Wende hin zu einer neoliberalen Gouvernementalität sprechen. Der Staat ist nun nicht mehr korrigierendes oder ausgleichendes ,Gegenstück' des Marktes, vielmehr werden die Logiken des (,freien') Marktes nun auch zu dessen zentralem Organisationsprinzip. Damit ändert sich auch die Art und Weise, wie Subjekte regiert werden. Mit der Figur des „Unternehmerischen Selbst“ verdeutlicht Ulrich Bröckling (2007), wie Subjekte im Kontext der neoliberalen Gouvernementalität dazu angehalten werden, über Selbsttechniken markt- und gewinnorientiert in sozialen Beziehungen zu agieren.

„Über diese Figur der unternehmerischen Freiheit zielt Regieren im Neoliberalismus darauf ab, dass die Subjekte selbstverantwortlich, risikofreudig und effizient ihre Selbstverhältnisse und Lebensweisen gestalten: Die Subjekte werden dazu geführt, ihre Lebensläufe über lebenslanges Lernen permanent zu optimieren, ihre Körper in Eigenverantwortung fit zu halten und ihre privaten Beziehungen effizient zu gestalten - alles im Namen und unter dem Versprechen der Freiheit.“ (Ludwig 2019: 3)

Angesprochen sind hier spezifische Weisen der Subjektivierung, die über die Anrufung der Freiheit zur eigenen Entfaltung funktionieren. Das unternehmerische 
Selbst verweist auf eine Regierung des Selbst die zur Unterwerfung des gesamten Lebens unter das Primat der Selbstoptimierung aufruft, wobei „das Ziel die Bewahrung der Reproduktion und Mehrung des eigenen Humankapitals“ ist (Opitz 2007: 105). Die Strategie der Responsibilisierung (also: bis ins Letzte für sich selbst verantwortlich zu sein, unabhängig vom Zugang zu entsprechenden Ressourcen, Rechten, Wahlmöglichkeiten etc.) wirft auch die brisante Frage auf, inwieweit - ursprünglich oder nach wie vor emanzipatorisch gedachte - Forderungen nach Selbstbestimmung das neoliberale Credo des Selbstmanagements ,bedienen'. Dieser Aspekt hat sich im Kontext des REVERSE-Projektes für die Fallstudie zu Mutterschaft als besonders relevant erwiesen, aber auch in den Fallstudien zur Ehe für al$l e$, zu Ethnisierung von Sexismus und zu Sexualpädagogik.

Wenn wir nun die derzeitige neoliberal-gouvernementale Regierungsrationalität der (Selbst-)Responsibilisierung mit einem veränderten Sexualitätsdispositiv zusammendenken, dessen Imperativ und Anrufung ebenfalls, die Gewährung der Freiheit' ist, so können wir fragen, ob ,Freiheit' auf diese Weise nicht zum autoritären Diktum wird. Alles, was diese autoritär gefasste ,Freiheit` (vermeintlich) einschränken könnte, wird dann zur Negativfolie: Die rassifizierten ,Anderen', die ,unfreien` Mütter, die ,moralischen' Feministinnen und die ,Ewig-Gestrigen', die ,immer noch` auf Ungleichheiten hinweisen. In der hier ironisch referierten Logik sind sie es, die der, eigentlichen Freiheit' im Wege stehen. Diejenigen, ,die sich nicht zufriedengeben', werden damit zu Störenfrieden der, eigentlich doch schon erreichten' und deshalb nur noch zu verteidigenden ,Freiheit'. Warum autoritäre Politikformen und Kräftefelder - wie sie in den gegenwärtigen Antifeminismen deutlich werden - genau hier , andocken', könnte also über die spezifische Modalität zeitgenössischer Subjektivierungen erklärt werden. Allerdings lässt sich mit diesem Zugriff die beobachtbare affektive, psychische Dynamik noch nicht unbedingt verstehen.

Wenn es darum geht die Frage zu klären, wie das Subjekt, dessen ,Selbstbestimmung' so vehement reklamiert wird, das aber gleichzeitig ,Andere' ausgrenzt sowie eigene Anteile ,abwehrt', in seinen Handlungsweisen konzeptualisiert werden kann, braucht es noch andere Zugänge. Damit ist ein Bogen geschlagen zu der eingangs bereits angesprochenen Frage der Vermittlung von Gesellschaftlichem und Psychischem. Um dieser Spur zu folgen, werden im Folgenden zwei weitere ,Lesarten` zur Geltung gebracht, die - wenn auch unterschiedlich - jeweils psychoanalytische Perspektiven auf Subjektivierungsweisen ${ }^{9}$ einnehmen. Zunächst wird in einer zweiten Lesart (4.), u.a. in Auseinandersetzung mit Judith Butlers Überlegungen zur Psyche der Macht (2001), kritisch reflektiert, inwiefern eine ,Logik der

9 Es sei hier daran erinnert, dass die Begriffe ,Subjekt` und ,Subjektivierung` in den verschiedenen Lesarten nicht genau dasselbe bezeichnen. 
Machtdiskurse“ ausreicht, wenn es darum geht Antworten auf antifeministische Mobilisierungen zu finden, die immer auch eine affektive Dimension haben. Danach wird mit der dritten Lesart (5.) eine weitere psychoanalytisch-gesellschaftstheoretische Perspektive mit Bezug auf Lacan ins Spiel gebracht.

\section{Psychoanalytische Perspektiven AUF SUBJEKTIVIERUNGSWEISEN}

Die Überlegungen der zweiten Lesart gehen davon aus, dass es im Hinblick auf das Verhältnis zwischen machtvollen Diskursen, Regierungsweisen und der Bedeutung der Psyche für die Subjektkonstitution viele offene Fragen gibt, die sich über machtanalytische Beiträge allein nicht klären lassen. Hier wird deshalb ein Verständnis dafür angeboten, wie Macht auch ,von innen heraus‘ wirkt. Das erscheint vor allem dann bedeutungsvoll, wenn es um subjektive (Ausdrucks-)Formen geht, die - trotz Politiken der Geschlechtergleichstellung - auf eine Beharrlichkeit traditioneller Geschlechterbilder und auch auf weiterhin bestehende geschlechterdifferente Handlungsmuster hinweisen, wie sie im Kontext des REVERSE-Projekts etwa in der Fallstudie zu Mutterschaft rekonstruiert werden konnten. Die im Rahmen der zweiten Lesart vorgeschlagene psychoanalytische Perspektive auf das Subjekt bezieht sich auf Diskurs, Erfahrung und Unbewusstes sowie gesellschaftliche Bedingungen gleichermaßen und schließt dabei den Körper in seiner ,Triebhaftigkeit‘ (im Sinne von ,Antrieb“ und ,Impulsen“) mit ein (Krüger-Kirn 2015). Nicht zuletzt über das Theorem der Intersubjektivität eröffnet sich ein Möglichkeitsraum, das Zusammenwirken innerer und äußerer Verhältnisse im ,verleiblichten Körper‘ als Spannungsverhältnis zwischen Anpassung und Ermächtigung wahrzunehmen.

\subsection{Zur Theoretisierung des Subjekts bei Freud}

Mit der Konzeptualisierung des Unbewussten entwirft Freud (1917a) eine grundsätzlich andere Perspektive als die Vorstellungen vom , aufgeklärten“ und ,vernünftigen' Subjekt. Die Differenzierung in ,bewusst' und ,unbewusst' geht mit einer Dezentrierung des Ichs einher, die im Sinne von Freuds Aussage: „Das Ich ist nicht Herr [...] in seinem eigenen Haus“ (Freud 1917a: 11) bis dahin geltende ,Gewissheiten` untergräbt. Freuds Konzept der Subjektwerdung stellt einen Zusammenhang zwischen Körpererfahrungen und dem Aufbau einer psychischen Struktur her, so dass das ,Ich als ein vor allem körperliches“ (Freud 1923b: 253) gefasst wird. Der Körper wird als Ort subjektiver Erfahrungen und psychischer Verkörperungen verstanden, also von Anfang an als ,Beziehungskörper' (vgl. de Clerck 2007). 
Neben der Theoretisierung des Unbewussten spielt in Freuds subjekttheoretischen Überlegungen die Unterscheidung von ,Sexuell' und ,Sexualität‘ eine zentrale Rolle. Eng damit verbunden ist das Konzept der Bisexualität, das mit der Vorstellung von der, Vielgestaltigkeit des Sexuellen“ ein breites Spektrum, eher: Kontinuum sexuellen Erlebens und Verhaltens aufspannt, dessen Grenzen fließend sind (Freud 1916/1917a: 331f). Mit der Unterscheidung zwischen ,Sexuell‘ und ,Sexualität' können beide Elemente nicht nur in einer produktiven Wechselseitigkeit gedacht werden, vielmehr lassen sich damit auch die im Diskurs ausgeschlossenen sinnlichen und libidinösen Aspekte des Sexuellen vom - alltagssprachlichen, medizinischen oder auch juridischen - Bedeutungshof der Sexualität abgrenzen und historisch situieren.

Freuds Idee eines grundlegenden Zusammenhangs zwischen dem Sexuellen (das auf den Körper verweist) und dem Unbewussten (als Element der psychischen Struktur) bringt sexuelle und soziokulturelle Ebenen in einen unauflösbaren $\mathrm{Zu}$ sammenhang, in eine spannungsvolle wechselseitige Beziehung, die das Konflikthafte in der psychischen Struktur ausmacht. Die Konfliktlinien zwischen dem Bewusstsein und dem Unbewussten, aber auch innerhalb des Unbewussten sind dynamisch wirksam und üben maßgeblichen Einfluss auf das Leben der Menschen aus. Das Begehren allgemein, wie jede Form der (geschlechtlichen) Identität, sind demnach von Grund auf konflikthaft, als lebenslang unabschließbarer Prozess angelegt und dem bewussten Zugriff weitgehend entzogen.

Als ,Theorie der unbewussten psychischen Prozesse“ vertieft eine solche (psychodynamische) Perspektive auf das Subjekt den Zugang zu den (gesellschaftlich und politisch vorfindbaren) Modi des Umgangs mit sich selbst und Anderen. Das Subjekt ist also, immer schon (in) Gesellschaft", und sein Unbewusstes über seine gesellschaftliche Bedingtheit strukturiert. Daher hängt die individuelle Subjekthaftigkeit eng mit der Ebene der sozialen Anerkennung zusammen, die hier nicht zuletzt mithilfe der psychoanalytischen Konzepte von Identifizierung und ,Abwehr ${ }^{10}$ reflektiert werden soll.

10 Aspekte, die im Rahmen der familialen und sozialen Interaktionserfahrungen abgewehrt werden müssen, sind im Unbewussten weiterhin existent. Sie ,drängen zu einer Wiederkehr', d.h. sie motivieren und beeinflussen auch weiterhin Handlungen und Interaktionen. Das Konzept der Abwehrmechanismen ist daher eng mit dem Konzept des Wiederholungszwangs verknüpft. Hierunter wird die Tendenz des Psychischen gefasst, die eingeprägten, unbewussten Muster psychischer Konflikte in aktuellen Beziehungen stets neu zu inszenieren. 


\subsection{Zur Frage der ,Abwehr“}

Als ,Abwehr ' bezeichnet die Psychoanalyse psychische Aktivitäten, die darauf abzielen, psychischen Schmerz in all seinen möglichen Formen (z.B. Angst, Scham, Entwertung) zu vermeiden. Abwehrmechanismen übernehmen in gewisser Weise die Funktion der Selbstregulation. Das heißt, dass jede Abwehr zugleich auf Erfahrungen verweist, die im Rahmen gesellschaftlich vermittelter Interaktionsprozesse nicht in das Selbstkonzept aufgenommen werden können und deshalb abgewehrt werden müssen. Als Kehrseite der bewussten Identität sind sie - entsprechend der psychoanalytischen Theorie des Unbewussten - als Nicht-Identität im Unbewussten weiterhin existent. Grundsätzlich sind Abwehrmechanismen unvermeidlich in die psychische Struktur eingeschrieben und dienen der (alltäglichen) Lebensbewältigung. Im Kontext subjekttheoretischer Überlegungen ist relevant, dass die - in einen intersubjektiven Prozess eingebetteten - Abwehrmechanismen, die Sagbares von Nicht-Sagbarem trennen, eine persönliche wie eine kulturhistorische Dimension haben.

Je nachdem, welche Abwehrmechanismen vorherrschen, sind deshalb nicht nur Rückschlüsse auf eine, individuelle Persönlichkeitsstruktur' möglich, sondern auch auf einen überindividuellen historisch-gesellschaftlichen Kontext. In diesem Sinne spiegeln individuelle Subjektivierungsweisen die mit den soziokulturellen Machtstrukturen intendierten Verdrängungsprozesse wider (Krüger-Kirn 2015: 127f).

Für die Frage der Mobilisierung bestimmter Affekte und Ressentiments im Kontext von Antifeminismen ergibt sich aus der Auseinandersetzung mit Abwehrmechanismen nun folgende Hypothese: In antifeministischen Diskursen werden ,verdrängte Anteile“ mobilisiert (also z.B. Erfahrungen, die zu seelischer Verunsicherung führen), die wiederum einen Rückgriff auf Abwehrmechanismen nahelegen. Welche Bedeutung dabei den kulturellen Vorstellungen von Geschlecht zukommt, vertieft Regina Becker-Schmidt (2017 [2007]) mithilfe der Frage, ob es einen Zusammenhang zwischen der frühen Ich-Entwicklung und der Abwertung des ,Weiblichen' gibt - und ob darüber ein Bezug zu Frauenfeindlichkeit und auch ,Fremdenhass' besteht. In ihrem kritischen Versuch sozial-, kultur- und subjekttheoretische Überlegungen zusammenzudenken, rekurriert sie auf Freuds Konzeption der Alterität, in der ,abgewehrte infantile Sexualängste, Misogynie und Xenophobie zueinander in Verbindung gesetzt werden“ (Freud 1987: 426ff, zit. nach Becker-Schmidt 2017 [2007]: 354). Freud macht hier, in seinem Entwurf einer Psychoanalyse (1895), auf die Ambivalenz gegenüber ,dem ersten Nebenmenschen“ aufmerksam, die damit zusammenhängt, dass das Kind die An- und Abwesenheit der Mutterfigur psychisch verarbeiten muss. In psychoanalytischer Sicht sind Wünsche nach Versorgung und eine Wut über erlebte Ohnmacht untrennbar mit der un- 
bewussten Repräsentanz der Mutterfigur verbunden. ${ }^{11}$ Ihre Abwesenheit impliziert ,Differenz' und markiert die psychische Aufgabe, zwischen Ich und Anderem/Anderen zu unterscheiden. Wenn Freud schreibt: „Am Nebenmenschen lernt der Mensch erkennen“ (Freud 1987: 426), legt er eine wichtige Spur zum Umgang mit ,dem Fremden'. Die psychische Herausforderung, den ,Anderen' nicht zu einem ,Fremden` zu machen, ist demnach konstitutiv mit der Anerkennung der eigenen Begrenztheit und der eigenen konstitutiven Abhängigkeit vom Anderen verknüpft.

Die psychischen Herausforderungen, die mit der Anerkennung der eigenen körperlichen Begrenztheit sowie der Anerkennung der Abhängigkeit vom/von Anderen im Sinne der konstitutiven, Angewiesenheit' des Subjekts auf Andere (und auch mit der Differenz zwischen ,Selbst" und ,Anderem') verbunden sind, machen eines der Kernthemen in der Entwicklung von psychischen Ich-Strukturen im Kontext der Subjektivierung aus. ,Der Andere' repräsentiert dabei, mit Lacan (1980) gesprochen, sowohl den ,mütterlichen /elterlichen und familialen Kontext, als auch - bzw. damit einhergehend - die je historisch gesellschaftlichen Bedingungen. ${ }^{12}$

Die Psychoanalyse beschreibt die Spannung zwischen Selbstbehauptung und Abhängigkeit und auch die Spannung zwischen Gleichheit und Differenz denn auch als eine lebenslang zu bewältigende (konflikthafte) Aufgabe. Auch wenn die psychodynamischen Konflikte, die ,den Anderen' zu einem ,Fremden` machen, sehr unterschiedlich sein können, so spielt hier - psychoanalytisch gesprochen - doch immer die Angst vor der Erkenntnis eine Rolle, dass die ,narzisstische Selbstbezogenheit' und ,Autonomie' eine Illusion ist, und diese Angst muss abgewehrt werden.

Die Kränkung, nicht autonom zu sein, gewinnt in dieser Lesart mit der ,Entdeckung der Geschlechterdifferenz' eine weitere Wendung und geschlechtsspezifische Färbung. Im Kontext der kulturellen Weiblichkeits- und Männlichkeitskonstruktionen wird der mütterliche Körper zum Bedeutungsträger von ,Abhängigkeit‘ und begründet - im Horizont einer bürgerlich-bipolaren Geschlechterordnung gedacht -

11 Auch die entsprechenden psychoanalytischen Konzeptualisierungen sind in spezifischer Weise historisch-gesellschaftlich situiert; das zeigt sich z.B. daran, dass die Erfahrung der ,Angewiesenheit‘ und Abhängigkeit anhand der Figur der Mutter lange Zeit unreflektiert als ,natürliche“ Bindungsfigur konzeptualisiert wurde.

12 Da die Worte immer zuerst von dem Anderen kommen, markiert die paradox anmutende Formulierung „Je est un autre/Ich ist ein anderer“ (Lacan 1980: 14) eben diese dem Menschen wesenhafte Subjektivierung durch die/den Andere/n. Gleichzeitig verweisen die Worte und die Bezeichnungen über den individuellen Erfahrungskontext hinaus auf den je spezifischen historischen und gesellschaftlichen Kontext der ,symbolischen Ordnung', die mit dem ,Namen des Vaters“ (,le-nom-de-père“) in Verbindung steht (vgl. Lacan 1980). 
geschlechterdifferente Ausprägungen der ,narzisstischen Wunde`, die mit der konstitutiven Abhängigkeit einhergeht. Während die Konfrontation mit der konstitutiven Abhängigkeit für die Tochter über einen Identifikationsentwurf der Ähnlichkeit mit der Mutter vermeintlich gelindert werden kann, bietet sich für den Sohn, der nicht über die Gebärfähigkeit der Mutter verfügt, als eine Möglichkeit der Kompensation der ,narzisstischen Wunde', eine Abwertung und Abwehr des mütterlichen Körpers an. Indem die - durch Verunsicherung ausgelösten - aggressiven Abwehrbewegungen auf die Mutter bzw. auf den ,Anderen' (rück-)projiziert und durch gesellschaftliche Geschlechterbilder gestützt werden, treffen sich ,Frauenhass' und ,Fremdenangst ${ }^{`}$. Kristeva (2001) macht hier auf den entscheidenden Zusammenhang aufmerksam, dass ,der Andere' nicht (nur) als ,ein Fremder' außerhalb von uns zu verstehen ist, sondern im Gegenteil als das Abgewehrte und ,Fremde“ im Subjekt selbst. Dieses Unbekannte, ,Befremdliche', das für jeden Menschen zugleich konstitutiv ist, widerspricht aber der Konzeption eines ,autonomen Subjekts“, das historisch, gesellschaftlich und epistemisch zugleich ,männlich“ gedacht ist. Insofern besteht eine Verbindung zwischen der ,Abspaltung', Verschiebung und Verdrängung der konstitutiven Abhängigkeit mit gesellschaftlichen Vorstellungen von ,Andersheit‘, die sich auf race, class und Gender gleichermaßen beziehen können.

Die Herausforderung und Fähigkeit mit dem Fremden in uns wie mit ,den Fremden von außen' umzugehen, verweist auf subjekt-, kultur- und gesellschaftskritische Aspekte gleichermaßen. Das betrifft prinzipiell alle Geschlechter, ist jedoch auch mit spezifischen Männlichkeits- und Weiblichkeitsdilemmata verbunden. Bezogen auf (lebensgeschichtlich kontextuierte) Selbstentwürfe rückt daher die Frage in den Vordergrund, welche unbewussten Konflikte sich artikulieren, wenn die Abwehr der ängstigenden und als bedrohlich erlebten Erfahrungen (mit Abhängigkeit, Geschlechtlichkeit etc.) als Verschiebung oder ,Projektion' auf ,den Anderen $\%$,den Fremden $\%$,das Weibliche' etc. bis in den gesellschaftlichen Raum hinein erfolgt - ein gerade im Kontext antifeministischer Mobilisierungen sehr verbreiteter und massiv in Erscheinung tretender Umgang mit eigenen Verunsicherungen. Eine psychische - und zugleich gesellschaftlich anerkannte - Form im Umgang mit Angst ist die oben beschriebene Abwehrform der Verschiebung (othering). So kann die Angst auf ,gefährliche Andere' projiziert, oder/und auf der diskursiven Ebene gar als ,Gefährdung des Sozialen` ausgegeben werden.

Hier zeigen Studien (z.B. Pohl 2004, 2010) wie rechtspopulistische Propaganda jene psychischen Konflikte und Abwehrmechanismen anzusprechen versucht, die mit der (realen und imaginierten) Veränderung des Geschlechterverhältnisses und der Erschütterung traditioneller Rollenbilder durch feministische Politiken und neoliberale Modernisierungen einhergehen können. In der rechtspopulistischen Propaganda werden diese Dilemmata projektiv als ,Krise der klassischen bürgerlichen Familie‘ bzw. als ,Krise der Männlichkeit‘ verhandelt. Die mit den Veränderungen von familialen Verwandtschafts- und (Für-)Sorgeverhältnissen unterstellte ,Auflö- 
sung der Zweigeschlechtlichkeit in eine Pluralität von Gender ${ }^{\star}$ mobilisiert dann nicht nur eine Angst vor dem Verlust einer imaginierten geschlechtlichen Eindeutigkeit und machtvollen Position innerhalb der Geschlechterhierarchie, sondern auch eine Angst vor dem Verlust der damit verbundenen familiären Strukturen. Insofern berührt der imaginierte Verlust einer patriarchalen Familienstruktur eine in die Tiefenstruktur der Gesellschaft eingeschriebene geschlechterbinäre Spaltung in Abhängigkeit vs. Unabhängigkeit. Die Frage nach der Mobilisierung affektgeladener Ressentiments bekommt hier eine weitere Wendung, insofern die hier aufgerufenen psychischen Bewegungen mit kollektiven gesellschaftlichen Allianzen einhergehen, die anschaulich machen, dass für eine Abwehr (im Sinne von Ab-/ Spaltung und Projektion) Vorstellungen von Ungleichheit Notwendigkeit und Bedingung zugleich sind.

\section{3 ,Psyche der Macht' und ,Macht der Psyche“}

Mit dem Konzept der „melancholischen Identifizierung“ gelingt es Judith Butler (2001), eine Verbindung zwischen kollektiver und individueller Abwehr zu formulieren und zu zeigen, wie Macht im Subjekt wirksam wird. Demnach wirken die Normen nicht einseitig auf die Psyche ein; vielmehr gilt es diejenigen Mechanismen begrifflich näher zu bestimmen und aufzuklären, die an Subjektivierungen im Sinne von ,kohärenten Identitäten' beteiligt sind - und über die ,auf der anderen Seite" auch subversive Bestrebungen denkbar werden. In ihre subjekttheoretischen Überlegungen bezieht Butler unbewusste Funktionsweisen der geschlechtlichen Subjektivierung mit ein und schlägt mit Bezug auf Foucaults Rede von der Produktivität der Macht eine Erweiterung vor. Im Vordergrund steht für sie zu klären, wie die Wirkungsweise der Macht, die als Voraussetzung für das psychische Funktionieren ausgemacht wird, sich innerpsychisch zu einer ,Subjektivationsnorm' konstelliert (Butler 2001: 95).

Dabei rekurriert Butler einerseits auf den Zusammenhang von Subjektivierung und Unterwerfung, der in Überwachen und Strafen (1976) von Foucault unter dem Begriff einer „den Körper einkerkernden Seele“ ausformuliert wurde (Butler 2001: 82; Foucault 1976: 42). Die Ambiguität des Begriffs ,Macht‘ verweist darauf, dass sich das Subjekt erst durch die Unterwerfung unter die Macht der Diskurse und Normen als ,autonomes Subjekt von Denken, Handeln und Fühlen' konstituieren kann. Um auf diese Doppeldeutigkeit hinzuweisen, bezeichnet Butler diesen Prozess in Anlehnung an Foucault als Subjektivation (Distelhorst 2009: 53f). Zugleich bezieht sie sich auf zwei Schriften von Freud, um den geschlechtlichen Identifizierungsprozess nachzuzeichnen: In Trauer und Melancholie (Freud 1916/17g) und Das Ich und das Es (Freud 1923b) beschreibt Freud einen Identifizierungsprozess 
im Kontext von nicht betrauertem Verlust. Eine Form den Verlust zu verarbeiten sei die, Wiederaufrichtung des verlorenen Objekts im Inneren des Subjekts'.

Mit Bezug auf die heterosexuelle Matrix spricht Butler von einer ,Kultur der Geschlechtermelancholie' (Butler 2001: 132) und folgert, dass jede Form von Geschlechtszugehörigkeit mit kulturell induzierten Verboten einhergeht. Geht es bei dem Verworfenen um die kulturell verdrängte, nicht eingestandene Homosexualität, „dann können wir mit einer kulturell vorherrschenden Form von Melancholie rechnen, die die Verinnerlichung der unbetrauerten und unbetrauerbaren homosexuellen Besetzungen anzeigt.“ (Butler 2001: 131) Entsprechend der Annahme, dass am Grunde jeder Identitätsbildung ein Verlust steht, wobei die Abwehr der nicht-lebbaren Anteile nicht nur die individuelle Subjektgeschichte (mit-)ausmacht, sondern auch durch kulturelle Identitätsvorstellungen motiviert ist, verfolgt Butler das Ziel gerade auch die politisch motivierten Ausschlüsse geschlechtlicher Identitäten zu dekonstruieren.

Wenn diese ,Verwerfungen' hingegen in einen historisch kontingenten Zusammenhang gestellt werden, so legt deren historische Bedingtheit im Umkehrschluss nahe, dass auch Veränderungen denkbar sind. Vor diesem Hintergrund erscheinen Körper und Subjekt nicht nur als diskursiver Ort intelligibler Identifizierungen, sondern auch als Ort intelligibler Abwehr. ${ }^{13}$ Tove Soiland merkt dazu - Butler zitierend - an, dass sie „der psychoanalytischen Annahme [...], dass das Subjekt auf der Grundlage einer Verwerfung entsteht“, zustimmt, allerdings „diese Verwerfung dennoch nicht für den sich entziehenden Fluchtpunkt des Sozialen" hält (Soiland 2010: 132).

Gegenüber Butlers Thesen von der Entstehung geschlechtsspezifischer IchStrukturen lassen sich aus psychoanalytischer Sicht einige Einwände vorbringen (vgl. dazu auch Soiland 2010: 7ff, 124ff; Becker-Schmidt 2017 [2007]: 339ff). An dieser Stelle soll vor allem der Zusammenhang von Körper/Sexuellem und Unbewusstem in den Fokus gestellt werden. Denn auch wenn sich Diskurse und normative Vorstellungen im Laufe der Geschichte verändern, Subjektwerdung daher immer mit veränderten Inhalten konfrontiert ist und auch mit unterschiedlichen Modi der Subjektivierung einhergeht, so lässt Butlers Subjektverständnis doch die Frage offen, wie eine Psyche ,ohne Körper' vorzustellen ist, zumal gerade dem Körper als ,Ort des Unbewussten` in der Psychoanalyse konstitutive Bedeutung zukommt. Anders formuliert: In Bezug auf die körperliche und psychische Subjektivierung bleibt

13 Ein Beispiel dafür ist Butlers Ansicht, dass das ,Subjekt‘ von der ,Psyche‘ zu unterscheiden sei (vgl. Butler 2001: 83): „Man muss unterscheiden zwischen dem Begriff der Psyche, der den Begriff des Unbewussten mit einschließt, und dem Begriff des Subjekts, dessen Bildung bedingt ist durch den Ausschluss des Unbewussten.“ (Butler 2001: 191, Fußnote 4$)$. 
bei Butler die Frage ungeklärt, wie die mit hegemonialen Identitätsentwürfen einhergehenden Verwerfungen und Ausschlüsse für das (leiblich-körperliche) Individuum konkret relevant werden, und zwar sowohl im Hinblick auf repressive wie auch auf produktive, gar widerständige Momente. Die fehlende Theoretisierung und Ausblendung der Faktizität des Körpers und der damit verbundenen bewussten und unbewussten Verkörperungen stellt in der Auseinandersetzung mit Butlers Körperund Geschlechtertheorien einen bedeutsamen Kritikpunkt dar.

Auch wenn sich Butler auf entsprechende kritische Einwände und Fragen kontinuierlich einlässt, so hält sie doch daran fest, Geschlecht als (ausschließlich) über ,Anrufungen' und Positionierungen hervorgebracht zu fassen. Sie geht weiterhin davon aus, dass sich geschlechtliche Positionen durch Bedeutungszuweisungen, $\mathrm{Zu}$ schreibungen oder Festschreibungen herstellen - und entsprechend auch verändern können. Dabei lässt Butler - so die in der zweiten Lesart vertretene Auffassung außer Acht, dass gerade auch die von ihr selbst aufgegriffenen ,Mechanismen der Identifizierung' psychodynamische Transformationsprozesse beschreiben, die keinesfalls nur auf linguistische Prozesse zu beziehen sind, sondern auch imaginärbildhafte und vor allem leibliche Ebenen miteinschließen. Selbst wenn ,Identifizierung' ausschließlich mit Über-Ich-Motiven (wie Bestrafungsangst, Zwangsidentifizierung etc.) in Verbindung gebracht wird, bleibt der libidinöse Aspekt der psychischen Mechanismen der Identifizierung (im Sinne einer leidenschaftlichen libidinösen Besetzung des Selbst und der Sehnsucht nach Akzeptanz und Zugehörigkeit) relevant.

Bei Butler rückt sowohl die Faktizität des geschlechtlich verfassten Körpers wie auch der leiblich gefühlte Körper (als Ort des Unbewussten mit wechselseitiger Einflussnahme auf die Subjektivierung) in den Hintergrund; psychoanalytisch betrachtet, wird so allerdings die potenziell subversive Kraft des Sexuellen verfehlt, die mit Freud nicht auf eine bestimmte Sexualität festgelegt ist und sich gesellschaftlichen Regulierungen auch widersetzt. ${ }^{14}$ Diese Einwände führen direkt zu der Frage, die Butler selbst aufwirft, wenn sie von ,abgewehrten Wünschen' schreibt, die ,durch die Zensur schlüpfen“ (Butler 2001: 138) und die auf, das Unmögliche im Möglichen' verweisen: Wie kann der Anteil, der sich identifiziert, oder der Anteil, der widersteht, theoretisch gefasst werden, ohne die Leiblichkeit und unbewusste Dynamik der Psyche begrifflich zu klären?

Eine Desomatisierung der Psyche reduziert die von Freud in den Diskurs eingebrachte Unterscheidung von ,Sexuell' und ,Sexualität" auf eine Wissenskategorie und geht mit der Gefahr einher, das dem Geschlecht Zugeschriebene mit dem ,Geschlecht an sich` zu verwechseln (vgl. dazu Rendtorff 1996: 13). Während Butler

14 Eine Befreiung aus der heterosexuellen Matrix droht dann mit einer Auflösung der Geschlechter- und Generationenordnung gleichgesetzt zu werden. 
die (melancholische) Identifizierung mit den zugewiesenen Geschlechtsvorstellungen als unausweichlich beschreibt, widerspricht die psychoanalytische Perspektive der Abwehr nicht nur einer endgültig festgelegten geschlechtlichen Identität, sondern fragt mit der psychoanalytischen Figur der ,Wiederkehr des Verdrängten', was das Abgewehrte (als sexuell-libidinöses Begehren) repräsentiert.

Eingebettet in die Strukturiertheit und Sinnhaftigkeit einer patriarchal-geprägten symbolischen Welt artikuliert die Figur der Abwehr und Wiederkehr des Verdrängten daher nicht nur gesellschaftliche Macht- und Geschlechterverhältnisse, sondern fordert dazu auf, das Abgewehrte genauer in den Blick zu nehmen. Wie bereits beschrieben, geht die psychische Dynamik der,Wiederkehr des Verdrängten“ im historischen Kontext der bürgerlich-bipolaren Geschlechterordnung für Jungen und Mädchen mit unterschiedlichen Aufgaben einher. Für beide aber führt eine ,Anerkennung der konstitutiven Abhängigkeit' und ,Entdeckung der Geschlechterdifferenz' an den Ort des Mutterkörpers; mit Hannah Arendt (1998 [1981]) gesprochen, zur Anerkennung der Natalität. Im Konzept des (männlich gedachten), autonomen Subjekts“ wird diese jedoch verleugnet; das ,spaltende' Subjekt, das sich auch selbst von der Tatsache des Geboren-Worden-Seins abspaltet, muss dann zu immer wirksameren Abwehrformationen greifen, je mehr diese Spaltung bedroht erscheint. Das psychoanalytische Konzept der Abwehr dekonstruiert demzufolge nicht nur die Idee der Unabhängigkeit und Autonomie als Phantasma der Omnipotenz, sondern verortet diese im Sinne einer psychischen Regression auf der Ebene des kindlichen Größenwahns, der die Anerkennung der Begrenztheit (nach Lacan die ,symbolische Kastration') verweigert.

Für die töchterliche Position gestaltet sich die Frage der Anerkennung des Mutterkörpers (bzw. der Natalität und ,ersten Differenz') vor dem Hintergrund gesellschaftlicher Geschlechterhierarchien zumindest ambivalent, zumal immer auch das ,kulturelle Erbe' der ,Minderwertigkeit des weiblichen Körpers' weitergegeben wird. Damit muss eine andere Art der narzisstischen Kränkung integriert werden; diese kann durch die symbolische Dimension von Mutterschaft (als Erzeugungsmacht des weiblichen Körpers) zwar in gewisser Weise kompensiert werden, zugleich ergibt sich hier aber auch das Problem der Reduktion auf die Gebärfähigkeit. Mit anderen Worten: Solange die Signifikation der Geschlechterdifferenzen dem männlichen Körper und Begehren eine Sprache geben, das Weibliche dagegen stumm bleibt, infiltrieren diese Zusammenhänge notwendigerweise immer wieder von Neuem jede weibliche Subjektivierung. Sie gehen unweigerlich in die Signifikationsprozesse zwischen Mutter und Tochter ein und geben den unbewussten Fantasien und Inszenierungen von Weiblichkeit eine bestimmte normative und verneinende Prägung.

Teresa de Lauretis widersetzt sich in ihrem Text Die andere Szene, Psychoanalyse und lesbische Sexualität (1999) der - einflussreichen - psychoanalytischen Auffassung von der ,Dominanz des Phallus' und dem damit verbundenen Bild der 
(symbolisch) ,kastrierten Frau', indem sie sich auf Freuds Einsichten in die ,Zerrissenheit des Ichs‘ (vgl. Becker-Schmidt 2017 [2007]: 358) als relative geschlechtliche Position zwischen ,normal' und ,pervers` bezieht. Über eine spezifische Neuinterpretation der psychoanalytischen Konzeption des Fetisch formuliert sie ein signifikantes Begehren auch jenseits des Phallus - in Bezug auf den eigenen/weiblichen Körper. Insofern der Fetisch zugleich auf das Wunschobjekt und dessen Abwesenheit verweist, kann das Begehren auf den weiblichen Körper - und zugleich auf die, durch die Abwehr unkenntlich gemachte, Sehnsucht nach dem eigenen Körper - gerichtet werden, und so letztendlich auch auf den fehlenden/ersehnten , anerkennenden Blick der Mutter‘. In diesem Verständnis ist eine logische Konsequenz, dass die Figur der ,Wiederkehr des Verdrängten“ als ,Kehrseite einer normativen Weiblichkeit' auch als Hinweis auf die aufgrund von Machteffekten aus der Sprache verworfenen Erfahrungen gelesen werden kann - was zugleich das Potenzial der Veränderung anzeigt.

Diese machtanalytische Perspektive markiert den fehlenden , anerkennenden Blick der Mutter' sowohl in einem praxiskritischen wie politischen Sinne als patriarchalen Zugriff auf Weiblichkeit. An dieser Stelle setzt auch die Kritik von Irigaray an, wenn sie die Position der Frau/Mutter innerhalb der patriarchal strukturierten Gesellschaft kritisiert und die strukturell-patriarchale Bedingtheit der symbolischen Ordnung unserer Kultur herausarbeitet. Innerhalb dieser Matrix der symbolischen Ordnung repräsentiert der Phallus das Signum des Begehrens. Es gibt dann kein weibliches Äquivalent zum Symbol des Phallus. Die ,Frage nach dem Ort der Frau' zeigt demnach die Leerstellen in der (patriarchalen) symbolischen Ordnung auf; demnach kann es auch im Unbewussten keine Signifikanz des Weiblichen geben. Irigaray zeichnet detailliert nach, dass die Bindung des Symbolischen an die ödipale Struktur nicht nur mit einer geschlechtlichen Asymmetrie einhergeht, sondern mit einer Verneinung des Weiblichen überhaupt (vgl. Irigaray 1979: 80, $188) .{ }^{15}$

Aus feministisch-psychoanalytischer Perspektive besteht die Herausforderung daher darin, normative Geschlechtlichkeiten in Bezug auf Mutterschaft und Vater-

15 Indem Lacan die symbolische Struktur ohne eine Symbolisierung der Weiblichkeit an die ödipale Position anlehnt, kann er seinen Anspruch, eine trianguläre Struktur zu denken, nicht wirklich einlösen. Auf diese Weise kann Lacan (laut Irigaray) auch zu keiner wirklichen Anerkennung von Differenz und Andersartigkeit finden. Soiland (2010) arbeitet bei Lacan eine Kontinuität bezüglich der Funktion des Weiblichen heraus, die sich von seinem Konzept des Frauentauschs nach Claude Lévi-Strauss über die sprachtheoretische Verschränkung des Ödipuskomplexes (die Macht des Phallus) bis hin zum weiblichen Genießen in „Encore“ (Lacan 1986) nachzeichnen lässt. Dies lege nahe, dass Lacan den Status der Frau als unveränderbar hinnehme (vgl. Soiland 2010: 238f). 
schaft nicht nur zu dekonstruieren, sondern zu beleuchten, dass der männliche Körper zum Eichmaß (Irigaray 1974: 70) erklärt wird. Damit wird markiert, dass der weibliche Körper, insbesondere in Bezug auf Mutterschaft, nur in seiner Funktion, nicht aber als eigenständiger und differenter Körper mit eigenen Erfahrungen in den gesellschaftlichen und wissenschaftlichen Diskurs aufgenommen und institutionalisiert wird. Das damit einhergehende Phantasma des Mütterlichen, das eine Kultur der Funktionalisierung und Fremdbestimmung des Weiblichen ermöglicht und perpetuiert, kann in dieser Lesart daher erst überwunden werden, wenn eine Anerkennung differenter Subjektivitäten (Irigaray 2010: 78) ein Denken sexueller Differenz und die fehlende Repräsentanz des Weiblichen in der symbolischen Ordnung der Geschlechter anerkennt und „damit unsere gewohnte [patriarchale, HK-K] Ordnung stört.“ (Irigaray 2013: 115)

\section{SUBJEKT DENKEN MIT DEM SPÄTWERK JACQUES LACANS}

Eine weitere Möglichkeit, die Subjekttheorie der Psychoanalyse für die Analyse von zeitgenössischen Antifeminismen, aber auch von anderen Autoritarismen fruchtbar zu machen, liegt darin, Überlegungen aus Jacques Lacans Spätwerk aufzugreifen und diese auf unsere Gegenwartsgesellschaft anzuwenden. Damit sind wir bei der dritten subjekttheoretischen Lesart in diesem Beitrag, die noch einmal eine andere theoretische Wendung vollzieht.

Feministische Theoretikerinnen wie Tove Soiland, Alenka Zupančič, Ida Dominijanni und Juliet Flower MacCannell haben in den letzten Jahren die Neuakzentuierung von Subjekt, Geschlecht und Begehren des späten Lacan für die Formulierung feministischer Gegenwartsdiagnosen aufgegriffen. Die Perspektive, die diese Denkerinnen für ein Verständnis der Widersprüche in aktuellen Geschlechterverhältnissen eröffnen, kann u.E. ebenfalls für die Frage produktiv gemacht werden, warum autoritäre Politik- und Protestformen derzeit erstarken oder genauer: was Subjekte für diese Angebote und Entwürfe überhaupt zugänglich macht.

Das Interessante an Lacans Spätwerk ist die darin formulierte These, dass sich für spätkapitalistische Gesellschaften ein folgenschwerer Wandel in den menschlichen Subjektivierungsweisen ereignet hat. Dieser Wandel steht für ihn im Zusammenhang mit einer neuen gesellschaftlichen Organisationsform des menschlichen Begehrens - und der spezifischen Rolle, die dem ,Genießen“ (jouissance) darin zukommt. Am deutlichsten drückt sich diese neue Organisationsform für Lacan in einer Neuausrichtung der Funktion des Über-Ichs aus. Das Subjekt wird hier also anders als in früheren Schaffensperioden (vgl. dazu die Ausführungen im vorigen Abschnitt) - historisch lesbar, und damit für politische Analysen anschlussfähig. 
Auch Lacans späte Subjekttheorie betont die Gesetze des Symbolischen, die für den Menschen sowohl einen unwiederbringlichen Verlust (an Unmittelbarkeit) als auch die Stiftung seines Begehrens - als produktive Kraft - bedeuten. Intersubjektivität bedeutet hier, dass Menschen grundlegend auf andere Menschen angewiesen sind und ihre Autorisierung und Anerkennung nur im Netz des Symbolischen erfahren. ${ }^{16}$ Mit dem Begriff des Genießens hebt Lacan nun noch etwas Zusätzliches hervor, das im intersubjektiven Bezug auf andere nicht gänzlich aufgeht. Die Reflexion über Subjekt und Subjektivierung wird damit nochmals neu ausgerichtet.

,Genießen“ ist ein Begriff, der jener Dimension angehört, die Lacan ,das Reale‘ nennt - eine Dimension, die zwar mit der Sprache entsteht, jedoch selbst nicht zur sprachlichen Ordnung gehört. Es handelt sich vielmehr um einen ,Anhang' oder ,Überschuss', der sich als Störfaktor in unserer sprachlichen Realität zeigt (Zupančič 2013). Das Reale ist jenes ,Mehr', so lässt sich mit Tove Soiland (2018: 101f) paradox formulieren, das von der Unmöglichkeit allumfassender Erfüllung ,übrig bleibt', damit also im Wesentlichen ein Unverfügbares, das an den Verlust gebunden ist, den Menschen als Preis für ihre soziale Existenz entrichten. Es ist keine Substanz, keine positiv beschreibbare Realität, sondern vielmehr etwas, das sich als Widerspruch zeigt, das auf das Ausgeschlossene und Nicht-Assimilierbare verweist.

Genießen meint daher notwendigerweise etwas anderes als Lust: Es bezeichnet eher den Kipppunkt, an dem „Lust [...] leicht ihr Gegenteil berühren kann“, ein „Gebiet des Exzesses“, ein ,zu viel“ (Rose 1982: 34, eigene Übersetzung). Es führt uns an eine bedrohliche Grenze des Symbolischen (in dessen Widerspruch oder Abgrund) und ist daher ,unerträglich, heimtückisch, zerstörerisch“ (Recalcati 2018 [2000]: 32f) - es drängt das Subjekt dahin, die Spaltung, die es symbolisch durchzieht, zu leugnen, zu überwinden, zu negieren. ${ }^{17}$

16 Das Symbolische bezieht sich im Werk von Lacan auf die Logik der Signifikanten, der Menschen als Sprachwesen unterworfen sind. Damit geht ein Verlust an Unmittelbarkeit einher, der letztlich für nichts anderes als für Abwesenheit steht, für etwas, das immer schon ,verloren' ist (oder genauer, für etwas, das im Nachhinein als Verlorenes gesetzt wird). Der Begriff „symbolische Kastration“ meint eine ursprüngliche Entfremdung, die das menschliche Begehren kennzeichnet, weil es nur durch Worte ausgedrückt werden kann bzw. sich im Netz der Sprache formt (vgl. Salecl 1994: 84).

17 Es gibt hier eine bedeutsame Wende im Denken von Lacan: Während das Begehrenssubjekt zunächst sehr stark an den Anderen gebunden war (und dort nach Anerkennung und Befriedigung suchte), ist es im Spätwerk sehr eng an das Genießen geheftet - und zwar an ein Genießen, „das sich nicht um den Anderen schert.“ (Recalcati 2018 [2000]: 42) Damit aber drängt es das Subjekt dahin, die Spaltung zu negieren, die es durch seinen Eintritt in die symbolische Ordnung unwiderruflich durchzieht. 
In der Realität begegnen wir dem Genießen lediglich in einer bestimmten Kategorie von Objekt, die Lacan Objekt a nennt: Es handelt sich um Objekte, die stark affektiv besetzt sind und die phantasmatisch auf eine Spur, auf einen Rest des Realen, auf ein Genießen verweisen. Das Objekt a, das Lacan die Objektursache des Begehrens nennt, ist daher jene ,phantasmatische Auskleidung“ (Soiland 2018: 103), in der sich das Genießen für uns ,zeigt‘ ${ }^{18}$

Wenn Lacan nun mit Blick auf den Spätkapitalismus von einem Wandel in der Subjektstruktur spricht, so bezieht sich dieser im Kern auf die historisch unterschiedliche Ausgestaltung der Beziehung zwischen Signifikant und Genießen. Erst die Formulierung des Objekt $a$ - das sich als eine Art Köder lesen lässt, der Genießen verheißt - erlaubt es ,historisch je unterschiedliche Weisen der Subjektivierung [anzunehmen], die sich in der Art unterscheiden, wie das Subjekt zu diesem Objekt angeordnet, $\mathrm{zu}$ ihm in Beziehung gesetzt wird (ist dieses Objekt verloren, zugänglich, demokratisch verteilt oder nur einem Herrn vorenthalten etc.).“ (Soiland 2018: 102) Damit kann nun gefragt werden, wie das Subjekt als Subjekt des Begehrens in jene Transformationsprozesse eingebunden ist, die Michel Foucault als Übergang der Souveränitäts- oder Disziplinarmacht zur biopolitischen Gouvernementalität beschrieben hat (vgl. die Ausführungen in der ersten Lesart).

Eine solche Analyse gibt ein Werkzeug an die Hand, subjekttheoretischen Sinn aus der paradoxen Formel aus feministischen Gegenwartsdiagnosen zu machen, dass - und wie - Subjekte heute durch Freiheit, Gewährung und Flexibilität regiert werden (vgl. dazu die Ausführungen in der ersten Lesart). Denn diese inzwischen häufig anzutreffende Beobachtung konstatiert, dass es gegenwärtig einen radikalen Gestaltwandel von ehemals emanzipatorischen Begriffen gibt, die in neue Herrschaftsformen transformiert oder zumindest eingebunden wurden (vgl. Maurer 2006; Sauer 2008; Dietze 2016; McRobbie 2010).

Mit Lacan lässt sich dieser Wandel als Übergang von einem ,Diskurs ${ }^{\circ 19} \mathrm{zu}$ einem anderen beschreiben (vgl. Soiland 2018; Grubner 2019). Den ersten nennt er

18 Es ist hier entscheidend, das Genießen nicht als etwas ,Originäres“ oder ,Vorkulturelles“ zu lesen. Das Genießen ist zwar ausgeschlossen, aber dennoch nicht, vorkulturell‘ oder ,vorsprachlich‘: Es entsteht mit unserer Ordnung, gehört ihr an, ohne sprachlich darin Platz zu finden.

19 Diskurs versteht Lacan hier als ,soziales Band“ und gibt ihm damit eine deutlich andere Ausrichtung als aktuelle diskurstheoretische Arbeiten. Ein Diskurs strukturiert nach Lacan unsere Wirklichkeit und es gibt keine außerdiskursive Realität. Diese Realität ist allerdings nicht komplett oder vollständig, sie ist kein Ganzes. Dies zum einen, weil es mehrere Diskurse gibt (Lacan unterscheidet vier, später fünf Diskurse). Und zum anderen, weil alle Diskurse einen Bezug zu einer Form des Genießens haben und damit auf 
,Diskurs des Herrn': Für diesen ist die Figur des autoritären Vaters charakteristisch oder, gesellschaftlich gesprochen, ein strenges Normensystem, das die Grenze zwischen Erlaubtem und Verbotenem festlegt: Eine Reihe von (meist männlichen) Expert_innen und Institutionen sorgt dafür, dass gut und böse, richtig und falsch klar unterschieden sind. Ärzte, Pädagogen, Politiker etc. sind nach dem Modell des strengen Vaters modelliert, der als Orientierungspunkt für die gesellschaftliche Ordnung fungiert. Die psychoanalytische Entsprechung für dieses Gesellschaftsmodell ist die klassische ödipale Situation: Freud beschreibt hier den Vater als jenen, dem die Aufgabe zukommt, ein Verbot auszusprechen. Damit trennt der Vater das Kind von der Mutter und richtet das Begehren des Kindes auf ein ,Außerhalb“ der Familie - auf die Gesellschaft.

Seit den 1970er Jahren ist nun das Wirken eines neuen Diskurses zu beobachten, der sich mit dem Lacan'schen Begriff, Diskurs der Universität‘ einfangen lässt: Der autoritäre Vater ist vom Thron gestürzt und zahlreiche gesellschaftliche Schranken sind gefallen: Der strenge Lehrer, der despotische Chef, der unerreichbare Universitätsprofessor usw. müssen einem Ideal weichen, das auf Egalität und Partizipation ausgerichtet ist. Lacan betont diesbezüglich bereits in den 1960er Jahren, dass durch die Liberalisierung die erhoffte Freiheit dennoch nicht errungen wird, dass sie nicht zum Untergang von Herrschaft und Hierarchie führt. Ihm zufolge ist nun ein anderer Meister auf der Bildfläche erschienen, der allerdings nicht mehr sichtbar ist. An die Stelle des Verbotes (das das Begehren orchestrierte) tritt nun ein Gebot: das Gebot, zu genießen.

Obwohl es von niemandem vorgeschrieben oder verlangt wird, geraten die Subjekte demnach unter eine Art Genusszwang, der mit ihren höchst individuellen Antrieben verschmilzt. Sie werden von einer inneren Stimme, von einem strengen und despotischen Über-Ich ,schikaniert, eingeschüchtert und provoziert [...], genau das zu tun, was der moralische Code unterdrückt oder verboten hat. ,Mach es einfach!" insistiert ein anonymer Unterton, sogar von T-Shirts und Autoaufklebern." (MacCannell 2000: 9, eigene Übersetzung) Auch die politische Führung, so zeigen Regierungschefs wie etwa Berlusconi oder Trump, ,identifiziert sich nicht mit Gesetz und Verbot, sondern mit Überschreitung und Genuss“ (Dominijanni 2014: 221). Dominijanni fügt hinzu, was das psychoanalytisch bedeutet: „Die symbolische Ordnung verschiebt sich von einer ödipalen symbolischen Ordnung zu einer postödipalen Unordnung" (ebd.).

Diese Verschiebung zeigt sich besonders deutlich darin, dass sich die Optimierung des eigenen Lusterlebens in eine soziale Pflicht verwandelt (Soiland 2013: 104). Der eigene Körper, die eigene Sexualität, Gesundheit, Ernährung, die eigenen 
Kinder sind sozusagen zu einer Angelegenheit emsiger Selbstverwirklichung geworden. Das Subjekt ist angehalten, alle sich bietenden Möglichkeiten auszuschöpfen, keine Chance zu vergeben. Einen Aspekt dieser Betriebsamkeit beschreibt der Kultursoziologe Andreas Reckwitz als Drang zur Selbst-Verbesonderung, der insbesondere die Mittelschicht umtreibe. Das „Streben nach Einzigartigkeit und Außergewöhnlichkeit“, so betont er, ist „nicht nur subjektiver Wunsch, sondern paradoxe gesellschaftliche Erwartung geworden“ (Reckwitz 2017: 9, Herv. i.O.).

Auf dieser Grundlage lassen sich zwei Thesen formulieren, zum einen in Bezug auf das gegenwärtige Erstarken autoritärer Politikformen und zum anderen hinsichtlich der widersprüchlichen Entwicklungen der Geschlechterverhältnisse zwischen Entdramatisierung und Re-Traditionalisierung (,Persistenz und Wandel'):

1. These: Die Hinwendung zu reaktionärem Autoritarismus und zur - für diesen charakteristischen - Projektion von Schuld auf Stellvertretungen (Sündenböcke) ist heute deshalb verführerisch, weil Feindkategorien einen äußeren Grund für das Scheitern am Genießen (an der eigenen Erfüllung, dem eigenen Glück) liefern.

Wie oft betont wird, spielen Sündenböcke in autoritären Politikformen eine ganz bedeutende Rolle. Insofern Ungerechtigkeit und Herrschaft in Zeiten der Globalisierung immer schwerer konkreten Urheber_innen zugerechnet werden können und damit weitgehend ihr Gesicht verlieren, wird die Schuld an strukturellen Benachteiligungen und Verelendungen zunehmend Feindkategorien zugeschoben. Diese können unterschiedlich gefüllt werden, etwa durch Feminist_innen, ,Multikultis' oder ,Flüchtlinge', die damit zu ,Ursachen' bzw. zu Schuldigen werden, an denen sich die Wut entladen kann (vgl. z.B. Fraser 2017).

Im Kontext der Überlegungen in der dritten Lesart geht es dabei vor allem um einen ,phantasmatischen Entzug': Die Sündenböcke dienen demnach dazu, eine ,äußere Schranke', einen Hinderungsgrund für das Nichterreichen des eigenen Genießens abzugeben; ein Genießen, das - obwohl unmöglich und unerreichbar - neoliberalen Subjekten in Aussicht gestellt ist. Damit lässt sich auch erklären, warum autoritäre Politikformen und Rechtspopulismus nicht nur bei Globalisierungsverlierer_innen auf fruchtbaren Boden fallen: Gerade auch Teile der ,emsig tätigen Mittelschicht', die den Kulturkapitalismus am Leben erhält, ringen mit dem neoliberalen Versprechen, dass Glück, Zufriedenheit oder innere Erfüllung zum Greifen nahe sind - und machen andauernd die Erfahrung, dass sie sich nie so wirklich einstellen wollen.

Diese Logik der Grenze hat Alenka Zupančič für die Ereignisse des 9. September 2011 auf den Punkt gebracht: Demnach hatte der Terror des 11. September oder vielmehr das, was daraus gemacht wurde - so etwas wie eine ,erlösende Funktion` in Bezug auf die erschöpfende Jagd nach dem persönlichen Glück. Der Kern 
der Botschaft lautete: „[E]s gibt etwas Objektives, das unserem Glück im Wege steht“ (2006: 176, eigene Übersetzung). Der Terror, der uns alle bedroht, ist die Grenze, die meinem Glück, meiner Entfaltung, meiner Freiheit von außen auferlegt wird. Ein wichtiger Effekt dieser Ver-Äußer(lich)ung (Externalisierung) zeigt sich erst auf den zweiten Blick: nämlich die beruhigende Gewissheit, dass das, was durch ,den Anderen' verunmöglicht wird, als weiterhin begehrenswert und grundsätzlich erreichbar bekräftigt wird. So erklärt sich für Zupančič die große Bereitschaft, tatsächliche Einschnitte sozialer und persönlicher Freiheiten in Kauf zu nehmen, wenn es um den ,Schutz vor Terror ${ }^{6}$ geht. ${ }^{20}$ Diese paradoxe Bereitschaft wird nur dann plausibel, wenn sie als Lockerung der Pflicht - des Über-IchDruckes - gelesen wird, das eigene Glück selbstverantwortlich herzustellen: „Die Pflicht fällt nicht länger mit dem Glück zusammen und niemand verlangt von uns, in Bezug auf den Terrorismus positiv zu denken und das Beste daraus zu machen.“ (Zupančič 2006: 176, eigene Übersetzung)

Es ließe sich die Frage stellen, ob die Nachwirkungen der ,Kölner Silvesternacht" (vgl. Bergold-Caldwell/Grubner in diesem Band) auf ähnliche Weise begreifbar werden: Aus subjekttheoretischer Sicht wird die Figur des ,Flüchtlings` als bevorzugtes Symbol der Begrenzung sichtbar. Sie wird dazu genutzt, eine schier unendliche Fülle an Problemen zu verkörpern: die Bedrohung , unserer Frauen', deren Freiheit sonst längst gewährleistet sei, die Aushöhlung des Sozialstaates, der sonst für alle Sorge tragen würde, die Konkurrenz auf dem Arbeitsmarkt, der sonst gerechte Jobperspektiven bereithielte, usw. Zugleich scheinen die Verbrechen an Europas Außen-Grenzen dem Selbstbild Europas als ,Hort und Wiege von Demokratie und Menschenrechten' anscheinend keinerlei Kratzer zuzufügen.

2. These: Die gegenwärtige Organisation des Genießens hat Auswirkungen auf das Verständnis von Emanzipation und wirft zugleich die Frage auf, wo Herrschaft heute sinnvoll angegriffen werden kann.

Vor dem Hintergrund der veränderten Subjektivierungsweise, die mit Bezug auf Lacan skizziert wurde, wird deutlich, dass sich gegenwärtige Antifeminismen nicht einfach als Backlash oder Rückschlag bisheriger Emanzipationsgewinne deuten lassen. Die - oft vorgenommene - Entgegensetzung zwischen (weiteren) Liberalisierungsforderungen und Forderungen nach Retraditionalisierung verdeckt vielmehr die Gemeinsamkeit in den verschiedenen Antworten auf den erheblichen ,Über-IchDruck‘, der durch die ,Verheißung der Erfüllung‘ (im Sinne von Vollständigkeit,

20 Zum Zeitpunkt der abschließenden Arbeit an diesem Beitrag im Frühjahr 2020 ließe sich hier auch ein Bezug zu den Entwicklungen und Verhältnissen im Kontext der CoronaPandemie herstellen, die ebenfalls als ,äußere Schranke' fungiert. 
Selbstverwirklichung, Selbstverbesonderung) ausgeübt wird. Sie werden damit als Identitätsversprechen erkennbar, die im Falle der Forderung nach Retraditionalisierung die Form eines Natur- oder Gottesgesetzes annehmen können, im Falle der Forderung nach (weiterer) Liberalisierung dagegen die Vorstellung von Machbarkeit und freier Wahl mit sich führen (vgl. dazu Uhlig/Rudel 2018; Soiland 2013).

Feministische Rückgriffe auf Lacans Spätwerk laden hier ein, darüber nachzudenken, auf welcher Ebene geschlechterhierarchische Strukturen heute privilegiert hergestellt und reproduziert werden, oder anders gesagt: was die Ursache für die hartnäckig weiterbestehenden Geschlechterungleichheiten ist. Sowohl in der Geschlechtertheorie als auch in den Politikfeldern von Gleichstellung und Unterstützung von Diversität gelten heute vor allem gesellschaftliche Normen und Zuschreibungen als Hemmschuhe für die progressive Veränderung hin zu einer Gesellschaft, in der vielfältige Identitäts-, Lebens- und Liebesformen nebeneinander bestehen können und anerkannt werden. Dass dies zu kurz greift, hat sich bereits mit der ,zweiten Lesart' deutlich gezeigt.

Lacans Subjekttheorie wirft nochmals pointiert die Frage auf, ob sich die Struktur geschlechtlicher Herrschaft heute tatsächlich in erster Linie über eine Normenkritik angreifen lässt. Ist die erfolgreiche Implementierung von Gender-Trainings und Diversitätssensibilisierungen in staatlichen Institutionen und wirtschaftlichen Unternehmen (noch) als Erfolg des Feminismus zu deuten - oder muss das Plädoyer für Entnormierung, für Offenheit und Flexibilisierung heute vielmehr als Teil einer Herrschaftslogik verstanden werden, die allerdings systematisch verdeckt bleibt? (Soiland 2013, 2014) Ist es dem (Spät-)Kapitalismus gelungen, sich auf die Freiheitsforderungen der Subjekte ein- und umzustellen, und so die darunterliegenden Strukturen der ökonomischen, patriarchalen und rassistischen Ungleichheit unangetastet zu lassen? Dass die politische Rationalität in spätkapitalistischen Gesellschaften auf starre Norm- und Rollenvorgaben durchaus verzichten kann, ist jedenfalls eine Beobachtung, die sowohl Foucaults Gedanken zur Gouvernementalität der Gegenwart als auch Lacans Subjekttheorie nahelegen.

Um hier geschlechtertheoretisch weiterzudenken, lenkt Tove Soiland (2018) die Aufmerksamkeit auf den Wandel vom ,Diskurs des Herrn` zum ,Diskurs der Universität‘. Es gibt hier etwas, das sich nicht verändert hat, nämlich die Idee, dass es allumfassende Erfüllung überhaupt geben könnte: Im ersten Fall (ödipales Modell) wird der vollumfängliche Genuss durch ein Verbot abgeschirmt. Dadurch wird er aber zugleich als etwas Mögliches aufrechterhalten, denn wenn der Vater/die Autorität es nicht verbieten würde, gäbe es ja Zugang dazu. Im zweiten Fall (postödipales Modell) wird der Genuss als etwas prinzipiell Zugängliches gesetzt: Jetzt haben alle die Möglichkeit Erfüllung zu erreichen - und sind selbst schuld, wenn diese sich nicht einstellt.

Es gibt nur eine Figur, die eine Entsprechung zu dieser Fantasie der Erfüllung darstellt: die (Fantasie der) Mutter als Inbegriff von Genuss, Befriedigung und 
Wohlbehagen, als ,Allgeberin“ oder Ort vollumfänglicher Lustbefriedigung. Es ist letztlich genau diese, Verheißung der Mutter', gegen die in der (patriarchal geprägten) psychoanalytischen Erzählung das ödipale Verbot errichtet wird. Mit dem späten Lacan wird diese ,ursprüngliche Verschmelzung' als etwas lesbar, das es nie gegeben hat. Sie ist vielmehr selbst eine rückwirkende Fantasie (oder ,Besetzung ${ }^{\natural}$ ).

Es drängt sich der Verdacht auf, dass der Verlust an Unmittelbarkeit, den die Psychoanalyse als Preis für die soziale Existenz thematisiert, selbst vergeschlechtlicht ist. Das bedeutet keineswegs, dass der Mangel oder Schnitt, der das Subjekt im Symbolischen charakterisiert, aus feministischer Sicht bestritten oder zurückgewiesen wird. Es geht vielmehr darum zu fragen, ob in der patriarchalen Subjektkonstitution nicht genau dieser Schnitt - also der Verlust, der Abhängigkeit generiert verleugnet wird. Für Soiland sind dies die zentralen Fragen, die Luce Irigaray an Lacan gerichtet hat, wenn sie behauptet, dass unsere Gesellschaft nicht zwei (oder mehrere) Subjekte hervorbringt, sondern ein männliches Subjekt und sein ,Anderes' - den vom ,Nicht-Männlichen' verkörperten ,Mangel', der somit die ,Infrastruktur` dafür abgibt, die Fantasie von Autonomie und Unabhängigkeit aufrechtzuerhalten (Soiland 2010: 157). Aus dieser Perspektive greift die feministische Kritik an binär codierten Geschlechterbildern zu kurz, solange sie den Blick nicht auf die Subjektkonstitution richtet, in die die Verleugnung des Mangels geschlechterhierarchisch eingebaut ist.

Das „,Ohne-Preis-Sein“ der Mutter“ (Soiland 2010: 159), der zugleich erhöhte wie erniedrigte, stets aber jenseitige Nicht-Ort, an dem ,die Mutter ' denkbar wird, ist daher ein zentraler Ort feministischer Kritik. Bis heute, so Soiland, hat die ,mütterliche Gabe' keine symbolische Existenz (Soiland 2016). Darauf wurde bereits mit der zweiten Lesart hingewiesen. Was hier gegeben wird ist sozusagen ,gratis verfügbar', wird als Naturressource behandelt und erfordert demnach auch keine Kompensation - im Gegenteil: Die ,mütterliche Gabe' muss unsichtbar, unerkannt und jenseits symbolischer Anerkennung bleiben, um ,für alle` dienstbar zu bleiben. ${ }^{21}$

In Rückerinnerung an die ,erste Lesart' könnte dies auch folgendermaßen ausgedrückt werden: Die Gesetze der Marktlogik, die heute insofern ,alles durchdringend" sind, als sie auch im Bereich des Persönlichsten und Intimsten eine möglichst rationale, effektive und kostenschonende Gestaltung anleiten, operieren heute zwei-

21 Vgl. zu diesem Aspekt auch die Arbeiten von Helga Krüger-Kirn (2017, 2019): In kritischer Auseinandersetzung mit der konstitutiven Bedeutung des Symbolischen für die menschliche Subjektivierung wird dort mit Bezug auf Julia Kristevas Konzept des abject (1982) und Bracha Ettingers matrixial border-space (2006) Subjektivierung im zwischenleiblichen schwangeren Erfahrungsraum von Mutter und Kind verortet und damit dem Mutterkörper ein Ort im Prozess der Subjektivierung zugewiesen. 
fellos ,geschlechtsneutral ${ }^{\star}$ - sie erfassen alle Geschlechter und Identitätsformen. Allerdings bleibt dabei notwendig ein ,Rest', etwas, das sich dieser Logik entzieht und die Form einer ,radikalen Alterität ${ }^{\star}$ annimmt. Dieser Rest, so die These, die von feministisch-psychoanalytischen Theoretikerinnen derzeit weiterverfolgt wird, ist die Figur der Mutter - und in diese Figur oder Fantasie ist das, was gemeinhin als ,weiblich' bezeichnet wird, nach wie vor eingeschlossen (vgl. dazu auch Hartmann 2019 und Hattinger-Allende 2019). Dieser Rest, diese ,Mater-Materie“ (Soiland 2010) muss als Ermöglichungsbedingung der um sich greifenden Verwertungslogik analysiert werden, ganz ungeachtet der heutigen Versuche, gerade auch den Bereich der Reproduktion und Fürsorge unter die Kontrolle der Rationalisierung und Professionalisierung zu zwingen.

Feministische Analysen, die Lacans späte Subjekttheorie aufgreifen, sind daher nicht zuletzt als Einladung zu verstehen, eine ,jenseits' oder ,unter' der Geschlechtsidentität (bzw. ihrer Festigung oder Dekonstruktion) liegende, zusätzliche Ebene der Geschlechtlichkeit in den Blick zu rücken und zu thematisieren. Diese ist keineswegs ,natürlich', sie wird allerdings auch nicht durch kulturelle Bedeutungszuweisungen hergestellt und sie legt keine sexuellen Ausdrucksformen fest. Sie handelt vielmehr von einer hierarchischen Spaltung im Herzen der Subjektkonstitution, die ,Männliches‘ und ,Nicht-Männlichem‘ trennt und damit die Leugnung von Unverfügbarkeit und Abhängigkeit perpetuiert. Dieser Spaltung nachzuspüren ist unter neoliberalen Bedingungen besonders wichtig, denn dies könnte die irreführende Alternative von ,progressiven' und ,rückwärtsgewandten' Kräften überwinden und demgegenüber die Idee radikaler Pluralität gesellschaftlich zur Geltung bringen.

\section{AntifEminismen IM SPIEgel VON SUbJeKtTHEORIEN - DER ERTRAG DER DREI LESARTEN}

Gerade in feministischer Perspektive wurde eine dezidierte Kritik an Vorstellungen von einem ,autonomen Subjekt' entwickelt, das sich - anscheinend klar abgegrenzt von anderen - aus sich selbst heraus entwickeln kann (vgl. z.B. Benhabib 1989; Benjamin 1989; Meißner 2010). Demgegenüber wird die grundsätzliche Angewiesenheit und Verletzlichkeit des Subjekts betont und eine wechselseitige Interdependenz der Menschen voneinander konstatiert. (Hier setzen auch die Debatten um Care und Reproduktionsarbeit an.) Diese grundsätzliche Angewiesenheit, die nicht nur im Kontext feministischer Reflexionen herausgestellt wird, erweist sich zugleich als brisant. Sie markiert ein konflikthaftes Moment, das in gewisser Weise auch abgewehrt werden muss, wobei die konkreten Abwehrmechanismen immer auch historisch-gesellschaftlich spezifisch und über Diskurse vermittelt sind. Die 
Dynamik der Abwehr ist vor allem im Zuge psychoanalytischer Theoriebildungen in ihrer Komplexität herausgearbeitet worden.

In Bezug auf die Verschränkung geschlechtertheoretischer und psychoanalytischer Perspektiven spricht Mechthild Bereswill (2018: 30) von einer ,doppelten Denkbewegung', mit deren Hilfe soziale Konstruktionen hinterfragt und zugleich die prägende tiefgreifende Auswirkung solcher Konstruktionen in ihrer Bedeutung für die Selbstentwürfe von Menschen thematisiert werden (können). Auch wenn ein produktiver Dialog zwischen den theoretischen Perspektiven der gegenwärtigen Geschlechterforschung und der Psychoanalyse - zumindest im deutschsprachigen Raum - bislang nur wenig erfolgt, zeigen sich - über alle Kontroversen und methodologischen Herausforderungen hinweg - doch auch Gemeinsamkeiten in den Subjekt-Verständnissen. Diese ergeben sich nicht zuletzt aus dem Anliegen (und der Herausforderung), die Spannung zwischen einer hegemonialen (Geschlechter-) Ordnung und deren ,Einfluss ‘ auf die Wertvorstellungen und Gefühle der Subjekte einerseits, und den , eigensinnigen Subjektivitäten“ andererseits zu fassen. Damit sind auch die Voraussetzungen, Kontexte und Modi der ,Subjektwerdung 'bzw. der ,Subjektkonstitution“ im Blick.

Mit der ersten Lesart wurde eine machtanalytische Perspektive ins Spiel gebracht, die den Blick auf (mögliche) Subjektpositionen in bestimmten MachtWissens-Ordnungen richtet und überdies den Zusammenhang von machtvollen Diskursen und Herrschaft aufzuzeigen vermag. Machtwirkungen entstehen demnach dort, wo sie einen bestimmten Möglichkeitsraum der Entfaltung hervorbringen und diesen zugleich in spezifischer Weise begrenzen. Damit kann nicht nur die Hervorbringung unterschiedlicher Subjektpositionen im Macht-Wissens-Diskurs verdeutlicht werden, sondern auch, welche unterschiedlichen Artikulationen sich damit verbinden (können). Zugleich werden über die historisch-genealogische Rekonstruktion Modi spätmoderner Subjektivierungen erkennbar, die über spezifische Selbst-Führungen erfolgen. Im Horizont der Studien zur Gouvernementalität der Gegenwart, die entsprechende ,Regierungskünste" herausarbeiten, wird deutlich, wie sich ,Freiheit‘ in ein Instrument der Einbindung in Herrschaftsverhältnisse verwandeln kann, das sehr schwer zu durchschauen ist.

Im Kontext aktueller Antifeminismen war und ist insbesondere auch die Frage von Belang, wie mit dem Fremden in uns selbst umgegangen werden kann. Hier sind subjekt-, kultur- und gesellschaftskritische Aspekte gleichermaßen im Spiel. Die Veränderungen in den Geschlechterverhältnissen und (Für-)Sorgeverhältnissen werden dabei häufig mit einer - als bedrohlich dargestellten (und von manchen subjektiv wohl auch empfundenen) - ,Auflösung der Zweigeschlechtlichkeit‘ in Zusammenhang gebracht - eine Vorstellung, die anscheinend nicht nur Angst vor dem Verlust einer imaginierten ,Eindeutigkeit von Geschlecht', sondern auch Angst vor dem Verlust der damit verbundenen familiären Strukturen mobilisieren kann. Hierfür hat die zweite Lesart Verstehensangebote gemacht. 
Die dritte Lesart hat die Neuakzentuierung von Subjekt, Geschlecht und Begehren des späten Lacan aufgegriffen, um Antworten auf die Frage zu finden, warum autoritäre Politik- und Protestformen derzeit erstarken und welchen Resonanzboden sie in neoliberalen Subjektivierungsweisen finden. Diese Perspektive formuliert den Vorschlag, gerade das, was das Subjekt als Intimstes empfindet - sein Begehren und Genießen - als genuin gesellschaftlich konstituiert zu denken und in eine kritische Gesellschaftstheorie einzubinden.

Gerade im Querbezug der Lesarten kann unseres Erachtens noch besser verstanden werden, dass - und wie - ,qua Geschlecht` heute auch durch Freiheit, Gewährung und Flexibilität regiert wird. Damit lässt sich zum einen ein erweitertes Verständnis des gegenwärtigen Erstarkens autoritärer Politikformen, insbesondere der ,Sündenbockpolitik، (Grubner 2019) gewinnen, und zum anderen kann ein anderer Blick auf die widersprüchlichen Entwicklungen der Geschlechterverhältnisse zwischen Transformation und Retraditionalisierung geworfen werden. Anhand subjekttheoretischer Überlegungen ließe sich u.E. auch besser verstehen, warum autoritäres antifeministisches und rassistisches Denken nicht nur bei jenen zu finden ist, die zu den Deprivilegierten und Entrechteten der gegenwärtigen politischen Ökonomie zählen, sondern auch bei denjenigen, die sich auf die neoliberalen Verheißungen als ,Leistungsträger_innen des Systems‘ eingelassen haben.

Die in diesem Beitrag diskutierten machtanalytischen Perspektiven auf aktuelle Modi der Subjektivierung ebenso wie die hier aufgegriffenen gesellschaftstheoretisch gefassten psychoanalytischen Subjekttheorien fordern u.E. dazu heraus, sowohl über die zeitgenössische Attraktivität autoritärer Protest- und Politikformen, als auch über die Verfasstheit aktueller Geschlechterordnungen - auch jenseits antidemokratischer Gesellschaftsentwürfe - auf neue Weise nachzudenken. Sie fragen nicht zuletzt danach, ob es zwischen diesen beiden Formen auch einen Zusammenhang gibt. Wir formulieren dazu abschließend einige Gedanken und markieren zudem inhaltliche Aspekte, die uns für den Kontext des REVERSE-Projektes besonders relevant erscheinen.

Verfolgt man aktuelle feministische Analysen, die über postfeministische Tendenzen im Verhältnis zur neoliberalen Ausrichtung zeitgenössischer Gesellschaften nachdenken, so weisen diese auf eine spezifische Dynamik hin. So spricht etwa Angela McRobbie (2010) von einem ,neuen Geschlechtervertrag“, der Frauen als Gleiche und Befreite zur Teilnahme in ehemals Männern vorbehaltenen Sphären einlädt und dennoch geschlechterhierarchische Strukturen in Ökonomie, Politik und Gesellschaft intakt lässt, deren systematische Kritik gar verunmöglicht. ${ }^{22}$ Aufgrund

22 Die Stärke der Analyse McRobbies ist, dass sie die damit verbundenen Machtwirkungen für unterschiedlich positionierte Frauen untersucht und in diesem Zusammenhang race sowie class herausstellt. 
des Wegfalls alter Beschränkungen und der Beteuerung, dass Gleichstellung doch längst erreicht sei, scheint ein Scheitern im Horizont des Gleichheitsprojektes nur an den Frauen selbst liegen zu können, die die ihnen gebotenen Chancen einfach nicht (angemessen) ergreifen. (Ein gutes Beispiel für die Strategie der Responsibilisierung, die die erste Lesart systematisch gekennzeichnet hat.)

Die mit der dritten Lesart aufgegriffene feministische Lektüre von Lacan verweist allerdings auf die grundlegend asymmetrische Signatur, die hier qua Geschlecht operiert. Demnach erfasst der spätkapitalistische Gestaltwandel der Subjektivierungsweise die Geschlechter keineswegs auf gleiche Weise; vielmehr behält die gegenwärtige Ordnung, die sich auf Gleichheit und Diversität beruft, ihre geschlechterhierarchische Struktur bei. Die „Illusion der Gleichheit“ (Campbell 2016: 245) bzw. die „rhetorische Modernisierung“ (Wetterer 2003), die Frauen heute als „Emanzipationsdarstellerinnen“ (Dietze 2017: 58) in Erscheinung treten lässt, hängt in dieser Sicht damit zusammen, dass Frauen heute zwei gleichermaßen unattraktive Optionen freistehen: Sie können ,entweder als männliche Subjekte oder als deren ,Anderes' in den sozialen Vertrag eintreten." (Campbell 2016: 245, eigene Übersetzung)

Gerade in der kritischen Auseinandersetzung mit Antifeminismen sollte jene herrschaftsförmige Geschlechterlogik analysiert werden, die sich , unterhalb ' der jeweils propagierten Geschlechterideale befindet. Diese Geschlechterlogik verbirgt sich nicht zuletzt in der Struktur spätkapitalistischer Begehrenssubjekte, die - ebenso wie ihre Vorgängermodelle - Patriarchales mit sich führt, insofern sie Mangel, Unvollständigkeit und Abhängigkeit auf jene auslagert, die Frauen genannt werden.

Die spätkapitalistische Organisationsform des Genießens, die Lacan beschreibt, setzt eine „dauerhafte Hektik des Subjekts“ (Recalcati 2019: 217) in Gang und schließt es in einen immer schneller werdenden Kreislauf der Jagd nach Erfüllung und der Produktion neuer Begehrlichkeiten ein. Die Mobilisierungskraft autoritärer Gedankenfiguren und ihre affektive Aufladung, sollte - so der Vorschlag der dritten Lesart - im Horizont dieser Zeitdiagnose verstanden werden. Denn Autoritarismen bieten anhand ihrer Freund-Feind-Schemata Projektionsflächen für das unvermeidbare Scheitern an, die ideale Lusterfüllung auf Dauer zu stellen. Feministinnen können in dieser Logik ebenso als Schuldige für den gesellschaftlichen Niedergang (oder als Störenfriede einer ansonsten intakten Ordnung) ins Visier geraten wie ,Multikulturalist_innen', Geflüchtete oder, die Elite'.

In der ersten Lesart werden solche Dynamiken über spätmoderne Subjektivierungen erklärt und in der zweiten Lesart vor dem Hintergrund individueller wie kollektiver Dynamiken der Abwehr reflektiert. Es geht hier nicht zuletzt um die psychische Fähigkeit einer grundlegenden Anerkennung von Differenz. Die in öffentlichen rassistischen/sexistischen Artikulationen zutage tretenden Affekte gehen nicht zuletzt auf eine Nicht-Anerkennung der (eigenen) Differenz zurück. Stattdessen findet eine (Ab-)Spaltung und Projektion statt, die über die hier eingenommene Per- 
spektive als spezifische Form der Abwehr kenntlich gemacht werden kann. Damit werden als unerträglich empfundene Spannungen nach außen gerichtet und projektiv (dem) ,Anderen' zugeschrieben. In Momenten krisenhafter Erschütterung wird dabei auf ein unbewusstes Abwehrsystem Bezug genommen, das sich aus individuellen und kulturellen Bildern zusammensetzt.

Im Kontext von Antifeminismen werden Transformationen der Geschlechterverhältnisse über Strategien der Polarisierung als destabilisierende gesellschaftliche Krisenszenarien entworfen. Der Inszenierung des Krisenhaften kommt dabei die Funktion zu, bisher latent vorhandene Ambivalenzen und Spannungen als manifeste Spaltung zu etablieren. Im Zentrum steht dabei eine Verhinderung der wechselseitigen Anerkennung durch Symbolisierungen, die sich durch rassistische, sexistische Inhalte und eine zugrunde gelegte hierarchische Geschlechterordnung auszeichnen, um damit eine Ent-Demokratisierung der Lebens- und Geschlechterverhältnisse zu legitimieren. Geschlechterpolitiken erscheinen ebenso wie sexuelle Politiken dafür als wichtiges Terrain, gerade weil sie ,das Private, Intime und Alltägliche ' der Menschen durchziehen.

Vor dem Hintergrund der Geschichte geschlechterhierarchischer Arbeitsteilung kann die gesellschaftliche Anerkennung und konkrete Organisation reproduktiver Tätigkeiten als Dreh- und Angelpunkt von Geschlechtergerechtigkeit gelten. Eine zentrale Voraussetzung für die Abwertung (und systematische Ausblendung) von Care(-Arbeit) stellt allerdings die mit der zweiten Lesart vorgestellte psychische und gesellschaftliche Abwehr der konstitutiven menschlichen Angewiesenheit auf ,den Anderen' dar. Damit wird zugleich die Position der Mutter abgewehrt. Ebenso wie in der dritten Lesart - wenn auch auf andere Weise - wird in diesem Zusammenhang auf die symbolische Ordnung Bezug genommen, in der der Platz der Mutter insofern ,leer' bleibt, als der Subjektstatus der Mutter unrepräsentierbar bleibt (Krüger-Kirn 2018). Insofern greift es zu kurz das Geschlechterverhältnis vor allem als Problem von Geschlechternormen, Rollenverhalten oder Geschlechterstereotypen zu verstehen. Auch der Blick auf soziale Ein- und Ausschlüsse greift zu kurz, wenn es darum geht, Geschlecht als Strukturkategorie zu fassen, die auf hierarchische Formen der Subjektivierung und Vergesellschaftung verweist. Gerade für die Analyse antifeministischer Argumentationen im Hinblick auf Familie und Elternschaft erscheint es unabdingbar, die dort vorgenommene Trennung der komplexen Zusammenhänge zu dekonstruieren.

Verwerfungen in Bezug auf die Position der Mutter kennzeichnen nicht nur eine patriarchal strukturierte symbolische Ordnung, sondern auch deren neoliberale/ neopatriachale (Campbell 2016; Soiland 2018) Ausprägung. Die im neoliberalen Diskurs prominente Behauptung, dass Frauen bereits autonome, aktive und selbstbestimmte Subjekte sind (Rutherford 2018; McRobbie 2010), verdeckt die weiterhin bestehenden Strukturen der Ungleichheit. Im Zuge der gouvernementalen Logik einer ,Führung durch Selbstführung' kann Fremdbestimmung überdies auf subtile 
Weise als Selbstbestimmung imaginiert werden. Dieser komplexe Zusammenhang wurde vor allem mit der ersten Lesart entwickelt.

Die vorgestellten Lesarten treffen sich nicht zuletzt an dem Punkt, an dem die Frage nach den Funktionsweisen von Herrschaft gestellt wird; sie gehen davon aus, dass das Soziale und das Subjektive keine voneinander abtrennbaren Realitäten sind, dass ,innen` und ,außen` vielmehr von einer gemeinsamen Logik durchzogen sind.

Allerdings unterscheiden sich die jeweiligen Diskursbegriffe (und damit auch die jeweiligen Subjektbegriffe) an einer entscheidenden Stelle: Während ,Diskurse bei Foucault genealogisch entstandene Macht-Wissens-Ordnungen sind, deren materielle Wirkung sich in Dispositiven zeigen kann, liegt das Augenmerk von Lacan auf jenen Aspekten, die in Diskursen nicht funktionieren, die nicht kontrollierbar und beherrschbar sind. Zwar untersucht Foucault Diskurse ebenso über die Brüche, Diskontinuitäten und Veränderungen in Epochen, sieht aber deren Konstellationen und Bezugnahmen zueinander als konstitutiv für eine historisch sich herauskristallisierende neue Ordnung. Während es bei Lacan um jene Risse geht, die dem Diskurs als Widersprüche inhärent sind (Klepec 2016: 121), hebt Foucault also die ordnenden Momente verschiedener Diskurse hervor. Subjekte werden in diesem Sinn immer wieder durch Diskurskonstellationen hervorgebracht, und ihre Selbsttätigkeiten unterliegen jeweils spezifischen historisch-kulturellen Techniken. Demgegenüber sind ,Diskurs` und ,Subjekt' bei Lacan um eine Unmöglichkeit, eine Unverfügbarkeit herum strukturiert, die Begehren sowohl stiftet als auch magnetisiert. Daraus folgt, dass ,[d]er politische Raum und konsequenter Weise die politischen Machtbeziehungen [...] auf dieselbe Weise dezentriert [sind] wie das Subjekt.“ (Tomšič/ Zevnik 2016a: 5f)

Demnach ist auch das Unbewusste als Teil des politischen Feldes aufzufassen. Feministische Denker_innen befragen darüber hinaus den Status des, Unverfügbaren'. Denn die Schwierigkeit des Subjekts, mit dem Unverfügbaren, auf dem es gründet - und damit mit Abhängigkeit und Verwiesenheit - zu Rande zu kommen, ist für sie kein neutrales Symptom, sondern bildet den Kern einer geschlechterhierarchischen Subjekt- und Gesellschaftsstruktur (das wird sowohl in der zweiten wie in der dritten Lesart reflektiert). Wenn also Begehrensformen zu historisch wandelbaren Bezugsgrößen einer kritischen Gesellschaftstheorie werden, so nicht primär deshalb, weil auch das Private oder Persönliche im politischen Feld Berücksichtigung finden muss, sondern weil Begehren und Genießen selbst gesellschaftlich strukturiert sind. 
Die historisch-genealogische Vorgehensweise Foucaults bietet, ebenso wie das Spätwerk Lacans ${ }^{23}$ und die kulturhistorische Perspektive Freuds (Psychohistorie), jedenfalls eine Sichtweise an, die Subjektivierung(en) als historisch veränderlich denkbar macht. Alle drei hier verfolgten Lesarten machen das - wenn auch auf unterschiedliche Weise - deutlich und erweisen sich damit nicht nur subjekttheoretisch, sondern auch gesellschaftstheoretisch als produktiv. Nicht zuletzt eröffnen sie weiterführende Reflexionshorizonte für die Frage, auf welcher Ebene die Enthierarchisierung und Entpolarisierung der gesellschaftlichen Geschlechterverhältnisse eigentlich ansetzen müssten.

\section{LITERATUR}

Arendt Hannah (1998 [1981]): Vita activa oder Vom tätigen Leben. 10. Aufl., München, Zürich: Piper.

Becker-Schmidt Regina (2017 [2007]): Pendelbewegungen - Annäherungen an eine feministische Gesellschafts- und Subjekttheorie. Aufsätze aus den Jahren 1991 bis 2015, Opladen/Berlin/Toronto: Barbara Budrich.

Benhabib, Seyla (1989): „Der verallgemeinerte und der konkrete Andere. Ansätze zu einer feministischen Moraltheorie“, in: List/Studer, Denkverhältnisse. Feminismus und Kritik, S. 454-487.

Benjamin, Jessica (1989): „Herrschaft - Knechtschaft. Die Phantasie von der erotischen Unterwerfung“, in: List/Studer, Denkverhältnisse. Feminismus und Kritik, S. 511-538.

Bereswill, Mechthild (2018): „Geschlecht als Konfliktkategorie und soziale Konstruktion. Überlegungen zu einer grundlegenden Spannung“, in: Bernd Ahrbeck/Margret Dörr/Johannes Gstach (Hg.), Der Genderdiskurs in der Psychoanalytischen Pädagogik. Eine notwendige Kontroverse (= Jahrbuch für Psychoanalytische Pädagogik 26), Gießen: Psychosozial-Verlag, S. 12-41.

Bergold-Caldwell, Denise/Grubner, Barbara (2017): „Ethnisierung der Geschlechterverhältnisse. Antifeminismus, Sexismus und Rassismus nach Köln“. Vortrag im Rahmen der Interdisziplinären Ringvorlesung des Zentrums für Gender Studies und feministische Zukunftsforschung, 22.06.2017. [http://media.blubrry. com/genderzentrum/podcast-kombinat.de/podcasts/gender-zentrum/rv-08-17-

23 Interessanterweise treffen sich Foucault und Lacan darin, dass sie beide sehr früh die Einschätzung vornahmen, dass die Studentenrevolte die erhoffte Befreiung nicht bringen würde, dass vielmehr das Ideal der Freiheit zu einer neuen Herrschaftstechnik avancieren könnte. Beide Denker haben offenbar bereits in den 1970er Jahren wichtige Aspekte der politischen Rationalität erkannt, die den heutigen Neoliberalismus kennzeichnet. 
bergold-caldwell-grubner-ethnisierung_der_geschlechterverhaeltnisse_antifemi nismus_sexismus_und_rassismus_nach_koeln.mp3; abgerufen am 27.05.2020]. Bergold-Caldwell, Denise/Grubner, Barbara (2020): „Effekte der diskursiven Verknüpfung von Antifeminismus und Rassismus. Eine Fallstudie zu Orientierungskursen für neu Zugewanderte“, in: Annette Henninger/Ursula Birsl (Hg.), Antifeminismen. ,Krisen'-Diskurse mit gesellschaftsspaltendem Potential?, Bielefeld: transcipt.

Bröckling, Ulrich (2007): Das unternehmerische Selbst. Soziologie einer Subjektivierungsform. 3. Aufl., Frankfurt a.M.: Suhrkamp.

Bröckling, Ulrich/Krassmann, Susanne/Lemke, Thomas (Hg.) (2007): Gouvernementalität der Gegenwart. Studien zur Ökonomisierung des Sozialen, Frankfurt a.M.: Suhrkamp.

Bublitz, Hanelore (2014): „Subjekt“, in: Kammler/Paar/Schneider, Foucault Handbuch. Leben - Werk - Wirkung, S. 293-296.

Bührmann, Andreas (1995): Das authentische Geschlecht. Die Sexualitätsdebatte der Neuen Frauenbewegung und die Foucault'sche Machtanalyse, Münster: Westfälisches Dampfboot.

Busch, Charlotte/Dobben, Brigitta/Rudel, Max/Uhlig, Tom David (Hg.) (2018): Der Riss durchs Geschlecht. Feministische Beiträge zur Psychoanalyse, Gießen: Psychosozial-Verlag.

Butler, Judith (2001): Psyche der Macht. Das Subjekt der Unterwerfung, Frankfurt a.M.: Suhrkamp.

Campbell, Kirsten (2016): „Political Encounters: Feminism and Lacanian Psychoanalysis“, in: Tomšič/Zevnik, Jacques Lacan. Between Psychoanalysis and Politics, S. 233-252.

de Clerck, Rotraut (2007): „Der zudringliche Blick. Sexualität und Körper. Subjektvorstellungen bei Sigmund Freud und Lucian Freud“, in: Philipp Soldt (Hg.), Ästhetische Erfahrungen. Neue Wege zur Psychoanalyse künstlerischer Prozesse, Gießen: Psychosozial-Verlag, S. 97-128.

Dietze, Gabriele (2016): „Das ,Ereignis Köln““, in: Feminina Politica 25 (1), S. 93 102.

Dietze, Gabriele (2017): Sexualpolitik. Verpflechtungen von Race und Gender, Frankfurt/New York: Campus Verlag.

Distelhorst, Lars (2009): Judith Butler. 1. Aufl., München: Fink.

Dominijanni, Ida (2014): „Das Gespenst der Sexualität. Frauen, Biopolitik und Neoliberalismus im heutigen Italien“, in: Feministische Studien 32 (2), S. 216-226.

Dreyfus, Hubert/Rabinow, Paul (1994): Michel Foucault. Jenseits von Strukturalismus und Hermeneutik, Weinheim: Beltz.

Ettinger, Bracha (2006): The Matrixial Borderspace, Minneapolis: University of Minnesota Press.

Foucault, Michel (1969): Wahnsinn und Gesellschaft. Eine Geschichte des Wahns im Zeitalter der Vernunft, Frankfurt a.M.: Suhrkamp. 
Foucault, Michel (1973): Die Geburt der Klink. Eine Archäologie des ärztlichen Blicks, Frankfurt a.M.: Suhrkamp.

Foucault, Michel (1976): Überwachen und Strafen. Die Geburt des Gefängnisses, Frankfurt a.M.: Suhrkamp.

Foucault, Michel (1993): „Technologien des Selbst“”, in: Michel Foucault/Rux Martin/Martin H. Luther/William Paden/Kenneth Rothwell/Huck Gutman/Patrik H. Hutton (Hg.), Technologien des Selbst, Frankfurt a.M.: Fischer Verlag, S. 2463.

Foucault, Michel (2001): Hermeneutik des Subjekts, Frankfurt a.M.: Suhrkamp.

Foucault, Michel (2003): „Die Gouvernementaltität“, in: Schriften in vier Bänden.

Dits et Ecrits - Band III. 1976-1979, Frankfurt a.M.: Suhrkamp, S. 796-823.

Foucault, Michel (2007): „Die Gouvernementalität“, in: Bröckling/Krasmann/Lemke, Gouvernementalität der Gegenwart, S. 41-68.

Foucault, Michel (2013): „Sexualität und Wahrheit - Band I: Der Wille zum Wissen“, in: Michel Foucault (Hg.), Die Hauptwerke. 3. Aufl., Frankfurt a.M.: Suhrkamp, S. 1021-1369.

Fraser, Nancy (2017): „Für eine neue Linke oder: Das Ende des progressiven Neoliberalismus“, in: Blätter für deutsche und internationale Politik 2, S. 71-76.

Freud, Sigmund (1916/1917a): Vorlesungen zur Einführung in die Psychoanalyse.

GW 11, Frankfurt a.M.: Fischer Verlag.

Freud, Sigmund (1916/1917g): Trauer und Melancholie. GW 10, Frankfurt a.M.:

Fischer Verlag, S. 428-446.

Freud, Sigmund (1917a): Eine Schwierigkeit der Psychoanalyse. GW 7, Frankfurt a.M.: Fischer Verlag, S. 3-12.

Freud, Sigmund (1923b): Das Ich und das Es. GW 13, Frankfurt a.M.: Fischer Verlag, S. 235-289.

Freud, Sigmund (1987): „Entwurf einer Psychoanalyse“, in: GW. Nachtragsband. Texte aus den Jahren 1895-1936, Frankfurt a.M.: Fischer Verlag, S. 375-488.

Gehring, Petra (2014): „Kommentar zu: Der Wille zum Wissen. Sexualität und Wahrheit Band I“, in: Clemens Kammler/Rolf Paar/Ulrich Johannes Schneider (Hg.), Foucault Handbuch. Leben - Werk - Wirkung, Stuttgart: J.B. Metzler Verlag, S. 85-92.

Gelhard, Andreas/Alkemeyer, Thomas/Ricken Nobert (Hg.) (2013): Techniken der Subjektivierung, München/Paderborn: Wilhelm Fink.

Graf, Julia/Ideler, Kristin/Klinger, Sabine (Hg.) (2013): Geschlecht zwischen Struktur und Subjekt. Theorie, Praxis, Perspektiven, Opladen/Berlin/Toronto: Barbara Budrich.

Grubner, Barbara (2019): „Autoritäre Demokratie. Über rechte Sündenbockpolitik und was sie mit Begehrensstrukturen zu tun hat", in: AEP-Informationen. Feministische Zeitschrift für Politik und Gesellschaft 46 (2), S. 55-58.

Hall, Stuart (1996): Critical Dialogues in Cultural Studies. Ed. By David Morley and Kuan-Hsing Chen, Routledge: London. 
Hartmann, Anna (2019): „Begegnung in der Differenz. Das ungelöste Problem der Sorge“, in: AEP-Informationen. Feministische Zeitschrift für Politik und Gesellschaft 46 (2), S. 16-18.

Hattinger-Allende, Sabine (2019): „Wider die Politik des Vergessens. Über schmerzhafte Einsichten und verheißungsvolle Versprechen“, in: AEP-Informationen. Feministische Zeitschrift für Politik und Gesellschaft 46 (2), S. 1215.

Irigaray, Luce (1974): Speculum. Spiegel des anderen Geschlechts, Frankfurt a.M.: Suhrkamp.

Irigaray, Luce (1979): Das Geschlecht, das nicht eins ist, Berlin: Merve Verlag. Irigaray, Luce (2010): Welt teilen, Freiburg/München: Verlag Karl Alber. Irigaray, Luce (2013): In the Beginning She Was, New York: Bloomsbury. Keller, Reiner (2011): Wissenssoziologische Diskursanalyse. Grundlegung eines Forschungsprogramms. 3. Aufl., Wiesbaden: Springer VS Verlag.

Kammler, Clemens/Paar, Rolf/Schneider, Ulrich Johannes (Hg.) (2014): Foucault Handbuch. Leben - Werk - Wirkung, Stuttgart: J.B. Metzler Verlag.

Klepec, Peter (2016): „On the Mastery in the Four ,Discourses““, in: Tomšič/Zevnik, Jacques Lacan. Between Psychoanalysis and Politics, S.115-130.

Kristeva, Julia (1982): Powers of Horror. An Essay on Abjection, New York: Columbia University Press.

Kristeva, Julia (2001): Fremde sind wir uns selbst, Frankfurt a.M.: Suhrkamp.

Krüger-Kirn, Helga (2015): Die konstruierte Frau und ihr Körper. Eine psychoanalytische, sozialwissenschaftliche und genderkritische Studie zu Körperpraktiken und Mutterschaft, Gießen: Psychosozial Verlag.

Krüger-Kirn, Helga (2017): „Ein Körper für Zwei. Schwangerschaft und Heterotopie“, in: Lea Spahn/Jasmin Scholle/Bettina Wuttig/Susanne Maurer (Hg.), Verkörperte Heterotopien. Zur Materialität und [Un-]Ordnung ganz anderer Räume, transcript, S. 199-213.

Krüger-Kirn, Helga (2018): „Care und Weiblichkeit“, in: Freie Assoziation 1/2018 „Familie und Geschlecht“: Psycho-Sozial Verlag, Gießen, S. 68-73.

Krüger-Kirn, Helga (2019): „Somatisches Wissen artikulieren. Annäherungen an die leiblichen Erfahrungen von Schwangerschaft und von Leihmutterschaft", in: feministische studien 1/2019, S. 49-66.

Lacan, Jacques (1980): Das Ich in der Theorie Freuds und in der Technik der Psychoanalyse. Das Seminar. Buch 2, Olten u.a.: Walter.

Lacan, Jacques (1986): Encore. Das Seminar, Band 10, Weinheim u.a.: Quadriga.

de Lauretis Teresa (1999): Die andere Szene. Psychoanalyse und lesbische Sexualität, Frankfurt a.M.: Suhrkamp.

Lemke, Thomas (2000): „Neoliberalismus Staat und Selbsttechnologien. Ein kritischer Überblick über die Gouvernementality Studies“, in: Politische Vierteljahresschrift 41 (1), S. 31-47. 
Lemke, Thomas/Krasmann, Susanne/Bröckling, Ulrich (2007): „Gouvernementalität, Neoliberalismus und Selbsttechnologien. Eine Einleitung", in: Ulrich Bröckling/Susanen Krassmann/Thomas Lemke (Hg.), Gouvernementalität der Gegenwart. Studien zur Ökonomisierung des Sozialen, Frankfurt a.M.: Suhrkamp, S. 7-41.

List, Elisabeth/Studer, Herlinde (Hg.) (1989): Denkverhältnisse. Feminismus und Kritik, Frankfurt a.M.: Suhrkamp.

Lorey, Isabel (2017): Immer Ärger mit dem Subjekt, Wien u.a.: transversal texts.

Ludwig, Gundula (2019): „Queer-feministische Perspektiven zur aktuellen Krise der Demokratie und der Autoritarisierung von Politik und Gesellschaft", Vortrag an der Philipps-Universität Marburg, 31.01.2019.

MacCannell, Juliet Flower (2000): The Hysteric's Guide to the Future Female Subject, Minneapolis-London: University of Minnesota Press.

Maurer, Susanne (2001): „Zentrierte Vielfalt? Zur Frage von Subjekt und Handlungsfähigkeit in der Auseinandersetzung mit poststrukturalistischem Denken“, in: Bettina Fritzsche/Jutta Hartmann/Andrea Schmidt/Anja Tervooren (Hg.), Dekonstruktive Pädagogik. Erziehungswissenschaftliche Debatten unter poststrukturalistischen Perspektiven, Opladen: Springer VS, S. 105-118.

Maurer, Susanne (2006): „Gouvernementalität, von unten her' denken. Soziale Arbeit und soziale Bewegungen als (kollektive) Akteure ,beweglicher Ordnungen““, in: Susanne Maria Weber/Susanne Maurer (Hg.), Gouvernementalität und Erziehungswissenschaft, Wiesbaden: VS Verlag für Sozialwissenschaften, S. 233-252.

Maurer, Susanne (2013): „,Subjekt‘ als Widerstand? Einige Annäherungen aus feministischer Perspektive“, in: Graf/Ideler/Klinger, Geschlecht zwischen Struktur und Subjekt. Theorie, Praxis, Perspektiven, S. 131-152.

McRobbie, Angela (2010): Top Girls. Feminismus und der Aufstieg des neoliberalen Geschlechterregimes, Wiesbaden: VS Verlag.

Menke, Christoph (2003): „Zweierlei Übungen. Zum Verhältnis von sozialer Disziplinierung und ästhetischer Existenz“, in: Axel Honneth/Martin Saar (Hg.), Michel Foucault. Zwischenbilanz einer Rezeption. Frankfurter Foucault-Konferenz 2001, Frankfurt a.M.: Suhrkamp, S. 199-210.

Meißner, Hanna (2010): Jenseits des autonomen Subjekts, transcript: Bielefeld.

Oldemeier, Anna Lena/Backöfer, Ferdinand/Maurer, Susanne/Aleksin, Katharina (2020): „Divergenz, Ambivalenz, Kongruenz. Verhältnisbestimmungen zwischen antifeministischem Diskurs und pädagogischem Feld“, in: Annette Henninger/Ursula Birsl (Hg.), Antifeminismen. ,Krisen'-Diskurse mit gesellschaftsspaltendem Potential?, Bielefeld: transcipt.

Opitz, Sven (2007): „Gouvernementalität im Postfordismus - Zur Erkundung unternehmerischer Steuerungsregime der Gegenwart“, in: Christina Kaindl (Hg.), Subjekte im Neoliberalismus, Marburg: BdWi-Verlag, S. 93-108. 
Pohl, Rolf (2004): Feindbild Frau. Männliche Sexualität, Gewalt und die Abwehr des Weiblichen, Hannover: Offizin Verlag.

Pohl, Rolf (2010): „Männer - das benachteiligte Geschlecht? Weiblichkeitsabwehr und Antifeminismus im Diskurs über die Krise der Männlichkeit“" in: Mechthild Bereswill/Anke Neuber (Hg.), In der Krise? Männlichkeiten im 21. Jahrhundert (= Forum Frauen- und Geschlechterforschung, Band 31), Münster: Verlag Westfälisches Dampfboot, S. 104-135.

Recalcati, Massimo (2018 [2000]): Der Stein des Anstoßes. Lacan und das Jenseits des Lustprinzips, Wien: Turia + Kant.

Recalcati, Massimo (2019): „Die Zerstörung des sozialen Bandes und die Hyperaktivität im Diskurs des Kapitalisten“, in: Limina. Grazer theologische Perspektiven 2 (2), S. 214-231.

Reckwitz, Andreas (2008): Subjekt, Bielefeld: transcript.

Reckwitz, Andreas (2017): Die Gesellschaft der Singularitäten. Zum Strukturwandel der Moderne, Berlin: Suhrkamp.

Rendtorff, Barbara (1996): „Geschlecht und Bedeutung. Über Verleugnung und Rückeroberung von Körper und Differenz", in: Barbara Rendtorff (Hg.), Materialität, Körper, Geschlecht, Frankfurt a.M.: Verein Sozialwissenschaftliche Forschung und Bildung für Frauen, S. 7-29.

Ricken, Norbert (2013): „Zur Logik der Subjektivierung. Überlegungen an den Rändern eines Konzepts“, in: Gelhard/Alkemeyer/Ricken, Techniken der Subjektivierung, S. 29-49.

Rose, Jacqueline (1982): „Introduction II“, in: Juliet Mitchell/Jacqueline Rose (Hg.), Feminine Sexuality. Jacques Lacan and the École Freudienne, New YorkLondon: Norton \& Company, S. 27-57.

Saar, Martin (2013): „Analytiken der Subjektivierung. Umrisse eines Theorieprogramms“, in: Gelhard/Alkemeyer/Ricken, Techniken der Subjektivierung, S. 17-29.

Said, Edward W. (1979): Orientalism, New York: Random House LCC US.

Salecl, Renata (1994): Politik des Phantasmas. Nationalismus, Feminismus und Psychoanalyse, Wien: Turia + Kant.

Sauer, Birgit (2008): „,Von der Freiheit auszusterben. Neue Freiheiten im Neoliberalismus?“, in: Marlen Bidwell-Steiner/Ursula Wagner (Hg.), Freiheit und Geschlecht. Offene Beziehungen, prekäre Verhältnisse, Innsbruck/Wien/Bozen: StudienVerlag, S. 17-32.

Schmid, Wilhelm (2000): Auf der Suche nach einer neuen Lebenskunst. Die Frage nach dem Grund und die Neubegründung der Ethik bei Foucault, Frankfurt a.M.: Suhrkamp.

Soiland, Tove (2010): Luce Irigarays Denken der sexuellen Differenz. Eine dritte Position im Streit zwischen Lacan und den Historisten, Wien: Turia + Kant. 
Soiland, Tove (2013): „Subversion, wo steckst du?“, in: in: Graf/Ideler/Klinger, Geschlecht zwischen Struktur und Subjekt. Theorie, Praxis, Perspektiven, S. 93114.

Soiland, Tove (2014): „Jenseits von Sex und Gender. Die sexuelle Differenz - Zeitdiagnostische Interventionen von Seiten der Psychoanalyse“, in: Anne Fleig (Hg.), Die Zukunft von Gender. Begriff und Zeitdiagnose, Frankfurt/New York: Campus, S. 97-125.

Soiland, Tove (2016): „Die mütterliche Gabe hat keine symbolische Existenz. Interview, geführt von Maya Dolderer", in: Maya Dolderer/Hannah Holme/Claudia Jerzak/Ann-Madeleine Tietge (Hg.), O Mother, Where Art Thou? (Queer-)Feministische Perspektiven auf Mutterschaft und Mütterlichkeit, Münster: Westfälisches Dampfboot, S. 203-213.

Soiland, Tove (2018): „Der Umsturz des Ödipalen. Ein feministisches Dilemma“, in: Busch/Dobben/Rudel/Uhlig, Der Riss durchs Geschlecht. Feministische Beiträge zur Psychoanalyse, S. 95-115.

Stoler, Ann Laura (1995): Race and the Education of Desire. Foucault's History of Sexuality and the Colonial Order of Things, Durham: Duke University Press.

Tomšič, Samo/Zevnik, Andreja (Hg.) (2016): Jacques Lacan. Between Psychoanalysis and Politics, Abingdon/New York: Routledge.

Tomšič, Samo/Zevnik, Andreja (2016a): „Introduction. Jacques Lacan Between Psychoanalysis and Politics“, in: Dies., Between Psychoanalysis and Politics, S.1-11.

Uhlig, Tom David/Rudel, Max (2018): „Wenn einem die Natur kommt. Das Identitätsversprechen im Antifeminismus", in: Busch/Dobben/Rudel/Uhlig, Der Riss durchs Geschlecht. Feministische Beiträge zur Psychoanalyse, S. 211-229.

Wetterer, Angelika (2003): „Rhetorische Modernisierung: Das Verschwinden der Ungleichheit aus dem zeitgenössischen Differenzwissen“, in: Gudrun-Axeli Knapp/Angelika Wetterer (Hg.), Achsen der Differenz. Gesellschaftstheorie und feministische Kritik, Münster, S. 286-319.

Zima, Peter V. (2017): Theorie des Subjekts. Subjektivität und Identiät zwischen Moderne und Postmoderne, UTB Verlag: Stuttgart.

Zupančič, Alenka (2006): „When Surplus Enjoyment Meets Surplus Value“, in: Justin Clemens/Russell Griff (Hg.), Jacques Lacan and the Other Side of Psychoanalysis. Reflections on Seminar XVII, Durham-London: Duke University Press, S. 155-178.

Zupančič, Alenka (2013): „Sexuelle Differenz und Ontologie“, in: Marlen BidwellSteiner/Anna Babka (Hg.), Obskure Differenzen. Psychoanalyse und Gender Studies, Gießen: Psychosozial-Verlag, S.131-149. 